\title{
A New Three-Dimensional Visualization System for Combining Aircraft and Radar Data and Its Application to RICO Observations
}

\author{
DAN K. ARTHUR AND SONIA LASHER-TRAPP \\ Department of Earth and Atmospheric Sciences, Purdue University, West Lafayette, Indiana \\ Ayman Abdel-Haleem, Nicholas Klosterman, And David S. Ebert \\ Purdue University Rendering and Perceptualization Laboratory, West Lafayette, Indiana
}

(Manuscript received 23 September 2009, in final form 22 December 2009)

\begin{abstract}
The analysis of diverse datasets from meteorological field campaigns often involves the use of separate 1D or combined 2D plots from various applications, making the determination of spatial and temporal relationships and correlations among these data, and the overall synthesis of information, extremely challenging. Presented here is a new 3D visualization tool, the Aircraft and Radar Data Collocation and Analysis in $3 \mathrm{D}$ (ARCA3D), that can combine data collected from different sources and at different scales, utilizing advanced visualization and user interface techniques, which allows for easier comparison and synthesis of such disparate data. The 3D tool is demonstrated with aircraft-based microphysical probe data and groundbased dual-polarization radar data all collected during the Rain in Cumulus over the Ocean (RICO) field campaign. The 3D volumes of radar data can be interactively selected and quantitatively probed, while aircraftmeasured variables can be viewed along the aircraft track plotted within the $3 \mathrm{D}$ radar volumes or plotted as time series within regions of interest relative to the radar echoes. The greatest benefits of the new software, the $3 \mathrm{D}$ viewing of large radar and aircraft datasets with user-driven controls, are difficult to communicate here in a static, 2D written medium, but the application of the tool toward a research problem is presented to elucidate the impacts of these benefits. The ARCA3D software is used to investigate the possible role of giant aerosol particles in the development of precipitation in trade wind cumuli. The temporal trends in the spatial location of the maximum differential reflectivity echoes within the clouds are examined with respect to the ambient giant aerosol number concentration and the measured cloud-base droplet number concentrations on 10 days. The results indicate that in trade wind cumuli of sufficient depth, giant aerosol may determine the original location of the earliest differential reflectivity maximum echo, and thus the first raindrops when present in higher number concentrations. However, when the giant aerosol are less plentiful, the number of cloud droplets activated above the cloud base may also play a role in determining the location of the earliest maximum differential reflectivity echo, and thus the earliest raindrops, in these trade wind cumuli.
\end{abstract}

\section{Introduction and motivation}

Meteorological field studies often provide researchers with diverse datasets gathered from different sources. The Rain in Cumulus over the Ocean (RICO) field campaign (Rauber et al. 2007) was one such study. Data were collected on trade wind cumuli using several platforms, including the National Center for Atmospheric Research (NCAR) S-PolKa radar (Keeler et al. 2000) and several

Corresponding author address: Dan K. Arthur, Dept. of Earth and Atmospheric Sciences, Purdue University, 550 Stadium Mall Dr., West Lafayette, IN 47907.

E-mail: dkarthur@purdue.edu aircraft on and around the islands of Antigua and Barbuda for several weeks in December 2004 and January 2005. The observational strategy of RICO was to gather data on shallow maritime convection and trade wind cumuli at a wide range of scales (Rauber et al. 2007). One goal of the project was to gain a better understanding of the warm-rain process, as traditional theory has been unable to explain how the growth of cloud droplets by condensation alone proceeds to drop sizes large enough to begin coalescence and produce precipitation as rapidly as has been observed (e.g., Paluch and Knight 1986; Beard and Ochs 1993; Rauber et al. 2007).

The use of separate applications for the examination of radar and aircraft data makes determination of 
correlations and synthesis of information between datasets quite difficult. During RICO, the NCAR C-130 aircraft sampled cloud dynamic, thermodynamic, and microphysical properties along one-dimensional transects through a field of clouds, whereas the S-PolKa radar gathered data on cloud and hydrometeor motion and evolution on larger scales. The C-130 aircraft measurements had a spatial resolution of roughly 4-100 m (depending on the sampling rate of the instrumentation and speed of the aircraft) while flying at constant altitudes typically for 30-60 min through a sector of the cloud field. These data exist on a GPS-based latitudelongitude coordinate system and are available as network Common Data Form (netCDF) files, with file sizes as large as $120 \mathrm{MB}$ each for $1-\mathrm{Hz}$ data from RICO (higher sampling rate data files are much larger). Time series plots of these aircraft data in 2D are often created with software specific to the aircraft platform. Radar measurements, on the other hand, are based in a polar coordinate system with spherical spatial resolution that decreases with distance from the radar site. For example, the half-power beamwidth of the S-PolKa radar used in the RICO field campaign is $0.91^{\circ}$ (Keeler et al. 2000), with 150-m range resolution between samples. The vertical resolution of the radar data and the temporal sampling rate depend upon the scanning strategy used; during RICO, this was typically 3-4 min for a full volume scan of a $90^{\circ}$ sector (Knight et al. 2008), resulting in 4-8 files per volume scan as large as 3-6 MB each. Although useful in their own right, separate $2 \mathrm{D}$ analysis software packages force an investigator to spend significant (and tedious) amounts of time trying to collocate the data and synthesize it into a conceptual picture before being able to use it to test hypotheses.

Presented here is a new visualization tool, the Aircraft and Radar Data Collocation and Analysis in 3D (ARCA3D), for analyzing such large multisource, multiscale datasets in three dimensions-the software also allows users to filter (select subsets of data) and/or search the combined datasets. This new tool facilitates data organization and synthesis by allowing the user to see all of the data at the same time, in three dimensions, and to filter out data that are not useful for the scientific problem being studied. It may be of use in both case studies of individual clouds or larger stormscale cloud systems, as well as in the analysis of the properties of an entire cloud field. To demonstrate its utility, the application of ARCA3D toward the effort of attaining a better understanding of radar echo evolution (and thus precipitation development) in small trade wind cumuli observed during the RICO field campaign is presented.

\section{Existing radar visualization tools}

A number of computer-based tools for meteorological radar data visualization have been developed over the years, ranging from specialized, research-oriented programs to generalized, commercial, multifunctional, operational application suites. Some of these applications have been the result of cooperative efforts between universities, research units within NCAR, and/or government and commercial organizations. The tools discussed herein all have advantages with regard to certain aspects of data visualization and/or analysis. Although some are capable of using data from multiple sources, few have the ability to use data simultaneously at the range of scales encountered in cloud microphysics. A review of radar data visualization tools is given here, and further discussion of the features of existing tools to motivate the development of ARCA3D is presented in section 3. This review is not intended to be an exhaustive examination but rather an illustration of the best techniques and capabilities of current radar visualization tools. ${ }^{1}$

Historically, meteorological radar visualization has evolved from simple monochrome oscilloscope-based range displays to either $2 \mathrm{D}$ plan position indicator (PPI) or constant altitude PPI (CAPPI) displays, with certain threshold values of different radar products (reflectivity, relative velocity, etc.) indicated by different shades of gray or different colors and/or 2D range height indicator (RHI) displays with similar gray or colored shading. A PPI display depicts the desired radar quantity at a given fixed elevation angle in polar coordinates of range from the radar versus azimuth angle. RHIs can be true vertical cross sections based on actual vertical scans or derived cross sections based on successive PPI scans (Rinehart 2004). Packages capable of radar visualization in $3 \mathrm{D}$ primarily have been based on the generation of isosurfaces. An isosurface is essentially a $3 \mathrm{D}$ surface indicating the extent of one or more selected values throughout a volume. More advanced rendering techniques have been and continue to be developed and will be discussed in this study. Desired characteristics in a new investigation tool include the following: a simplified graphical user interface (GUI) with real-time interactivity, simultaneous visualization of multiple datasets,

\footnotetext{
${ }^{1}$ Other applications reviewed (Arthur 2009) were PPI-MMM (Plan Position Indicator-Mesoscale and Microscale Meteorology), CEDRIC (Custom Editing and Display of Reduced Information in Cartesian Space), McIDAS-X (Man Computer Interactive Data Access System), GEMPAK/N-AWIPS (General Meteorology Package/National Centers for Environmental Prediction Advanced Weather Interactive Processing System), WDSS-II (Warning Decision Support System-Integrated Information), Gibson Ridge Software GRLevel3, GRLevel2 (and Analyst), and IBM Deep Thunder.
} 
quantitative output including flexible queries and/or filters of collocated data from multiple datasets, and 3D visualization without the use of isosurfaces.

The Solo II application (Oye et al. 1995), the integrated data viewer (IDV; Unidata Program Group 2009a), and the Visualization and Analysis Platform for Ocean, Atmosphere, and Solar Researchers (VAPOR; Clyne et al. 2007) software all have some real-time interaction capabilities. One of the more versatile radarspecific display packages, Solo II, allows simultaneous multivariable display in 2D from sweep files (a single file for each elevation angle during a continuous azimuthal sweep across the desired radar sector). Data can be edited graphically as well. Displays can be zoomed, panned, and advanced forward and backward through consecutive or nonconsecutive sweep files. A data widget displaying numerical values updates interactively when the user clicks the mouse cursor on different points in the data display. However, the display of different radar variables on separate panels, and the limitation of displaying only one 2D sweep at a time, requires the user to compare features by trying to locate a region of interest in different panels, making it difficult to identify features in other variables that might be collocated, above, or below a given sweep. This software only displays radar data; other types of data cannot be ingested into this package.

The IDV package, arguably one of the more flexible tools among current analysis software, allows researchers to create cross sections, profiles, animations, and value readouts from many different types of data. Radar data can be displayed by IDV as traditional PPI displays in $2 \mathrm{D}$ or $3 \mathrm{D}$, as a CAPPI display, as a $2 \mathrm{D}$ or $3 \mathrm{D}$ RHI, 3D volume scan, or a 3D isosurface. Most of these displays are interactive-the perspective of the image can be changed without entering new parameters and waiting for a new image to be rendered. The point of view can be panned, zoomed, or rotated via mouse movement and animation is also available. A data probe similar to the Solo II data widget is also available to display numerical values as well as $2 \mathrm{D}$ time series of multiple fields at a single point. The recently developed VAPOR package from NCAR, although not specifically a radar visualization tool, is an implementation of several newer user interface and 3D visualization techniques. A data probe is available as a $2 \mathrm{D}$ vertical cross section. Controls for the data probe in both IDV and VAPOR, and the quantitative display of the data within the probe, are available for real-time user interaction.

In addition to the 3D capabilities in the applications discussed earlier, the University of Wisconsin-Madison Space Science and Engineering Center (SSEC) developed the Vis5D system in 1988, and this has been superseded by Vis5d+ (Space Science and Engineering
Center 2009). The Vis5d+3D displays use isosurfaces to represent threshold values. Isosurface rendering is one of the major 3D visualization techniques used for meteorological data. A primary advantage of isosurfaces has been speed and interactivity along with the comprehension of the three-dimensional structure of weather phenomena (Carneiro et al. 1996). Isosurfaces can be drawn entirely in one color or colored according to the values of another variable (Hibbard et al. 2009). Mouse movement is used to control perspective interactively. Multiple thresholds can be chosen and opacity varied so that inner values are visible.

Isosurfaces also have some disadvantages, however, such as lack of fine detail and flexibility during rendering, and some ambiguity issues (Carneiro et al. 1996), especially when an investigator desires to see a complete picture of widely varying data. An unfortunate choice of isosurface value(s) can lead one to miss a particular feature within a cloud that might be useful for understanding its behavior. The Vis $5 \mathrm{~d}+$ package is also capable of limited volume rendering with radar data. This is a technique for displaying a 3D field as a semitransparent colored fog, in an attempt to view several isosurfaces at once and decrease the likelihood of missing features in the data. Each value is still depicted as an isosurface; however, even though Vis $5 \mathrm{~d}+$ calculates the array to be rendered as a $3 \mathrm{D}$ volume, the visualization technique is still limited to isosurfaces (McCaslin et al. 2009). The VAPOR package from NCAR can display isosurface and volume rendering in 3D (Clyne et al. 2007). The VAPOR application was designed as a general geophysical visualization tool, with the ability to run on commodity personal computer hardware. It has not been used specifically for radar visualization, but it offers many promising features for qualitative and quantitative exploration of large amounts of data in 3D.

The tools discussed previously each have certain strengths with regard to visualization of radar data. The advantages of the ARCA3D package, described in detail in the next section, include many features that overlap those described earlier, along with the ability to work with large amounts of radar and aircraft data simultaneously, advanced 3D visualization techniques, and flexible data selection.

\section{Description of the new software}

For microphysical research applications, isosurfacebased 3D products can present serious drawbacks. Specific values of multiple parameters in the 3D domain are difficult to obtain from isosurface representations, and navigation of the 3D space, particularly when viewing multiple translucent surfaces, can be disorienting and confusing without clear reference coordinates. Overcoming 
the challenge of collocating and synthesizing microphysical data with larger-scale radar data is one goal of the development of ARCA3D. Previous development work on collocating radar and aircraft data specifically has been limited to time series displays, which do not provide the investigator with the spatial relationship between radar samples, between radar and aircraft data (e.g., Ellis et al. 2001), or to 2D visualizations, which do not provide a complete context for both datasets.

Although the new system can render 3D visualizations of two datasets on different coordinate systems independently, to determine collocation of the polar coordinate radar volume with the latitude-longitude-altitude coordinate aircraft data the latter coordinates are used along with altitude and range from the radar site of each radar sample to calculate geodesics (Vincenty 1975), obtaining latitude-longitude coordinates for each radar sample.

The ARCA3D system ${ }^{2}$ uses netCDF data (Unidata Program Group 2009b) with Climate and Forecast metadata (Lawrence Livermore National Laboratory 2009) to maintain standardization and transferability. Radar data and aircraft data files must be in netCDF format for ARCA3D, and for the former a file translator such as that used with the Solo II software package (called xltrsii) can be used to convert the radar data files to netCDF.

\section{a. Software control and navigation}

Within ARCA3D, the datasets can be queried to look for correlations between aircraft microphysical probe variables and radar data when they are spatially and temporally collocated. Such correlations can be used to make inferences across the a field of cumuli, for example, where radar echo histories of clouds may be well documented but microphysical probe data are lacking. Any single radar variable and multiple $1 \mathrm{D}$ aircraft variables can then be visualized simultaneously. Data are loaded into the visualization system in a manner depending on data type. Radar data are grouped into sets of individual (converted) sweep files corresponding to volume scans. The user can then navigate through volume scans sequentially or nonsequentially. Aircraft data are read as a single file, which may span a period of many radar volumes depending on flight/file duration.

The system uses a transfer function scheme to plot data quantitatively by color and/or opacity (Fig. 1). The use of opacity in a volume-rendering system allows an in-

\footnotetext{
${ }^{2}$ At present, the software is Windows based, and the current study was performed on a Dell Precision Workstation 690 with dual-core Intel Xeon 3-GHz CPU, Microsoft Windows XP Professional, 2-GB RAM, and nVidia EVGA e-GeForce 8800 Ultra 768-MB graphics card, and MATLAB 7.4.0.287 (R2007a).
}

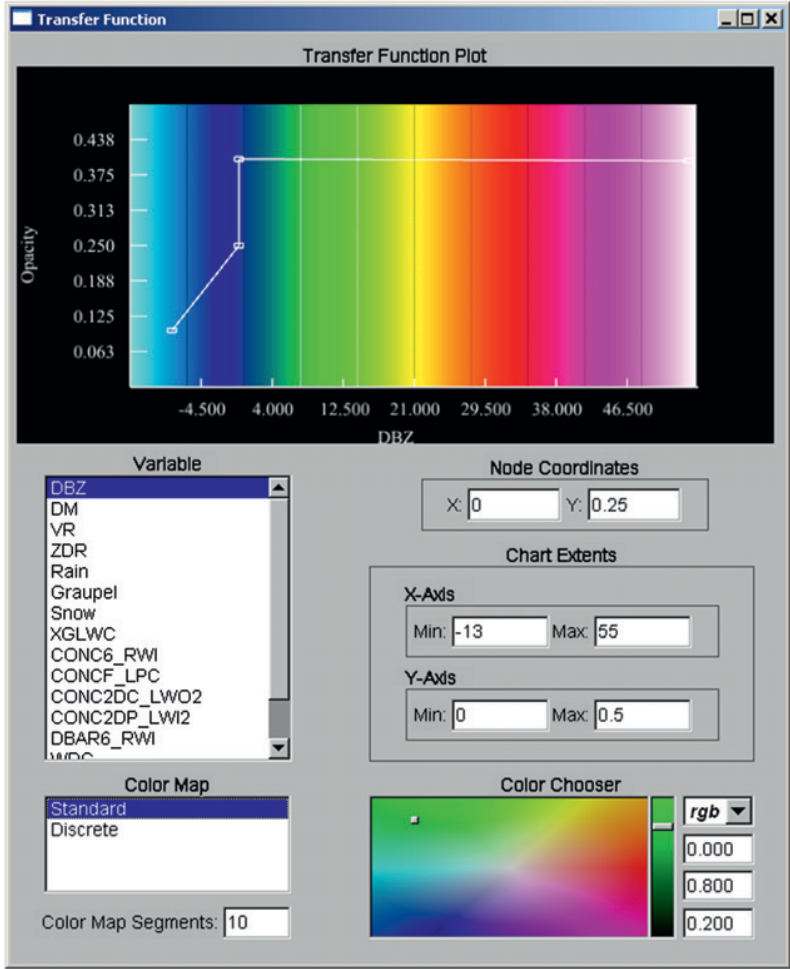

FIG. 1. Transfer function interface for converting numerical data into color and opacity values for 3D visualization in ARCA3D. The units of the selected data variable are along the abscissa and a fraction of opacity is along the ordinate. The function shown displays $Z_{e}>0 \mathrm{~dB} Z$ at $40 \%$ opacity, decreasing from $25 \%$ to $10 \%$ opacity with $Z_{e}$ decreasing from $0 \mathrm{~dB} Z$ to $-5 \mathrm{~dB} Z$. Nodes in the transfer function and colors in the desired color map can be edited numerically for precise definition.

vestigator to view more data at one time in $3 \mathrm{D}$ than would be visible through the use of a limited number of isosurfaces at set values. The user can set limits on what range(s) of values can be viewed by controlling the values of the transfer function. For example, the edges of a cloud on radar could be made visible but more translucent than more dense areas of the cloud with higher reflectivity values, allowing a user to see "through" the cloud edges to other possibly significant cloud features within the cloud but still see those edges to know the cloud size or where the feature is located within the overall cloud. A separate transfer function can be defined for each variable in the combined data as well as for derived quantities such as hydrometeor type (hail, graupel, snow, rain, etc.).

Two 3D views are available simultaneously (Fig. 2), which the user can configure for different radar variables or different perspectives of the same variable. Using mouse and keyboard, the operator can rotate, pan, and zoom within the $3 \mathrm{D}$ view to examine any particular region more clearly. Coordinates can be input to define the data that is displayed in $3 \mathrm{D}$ to be within desired absolute 

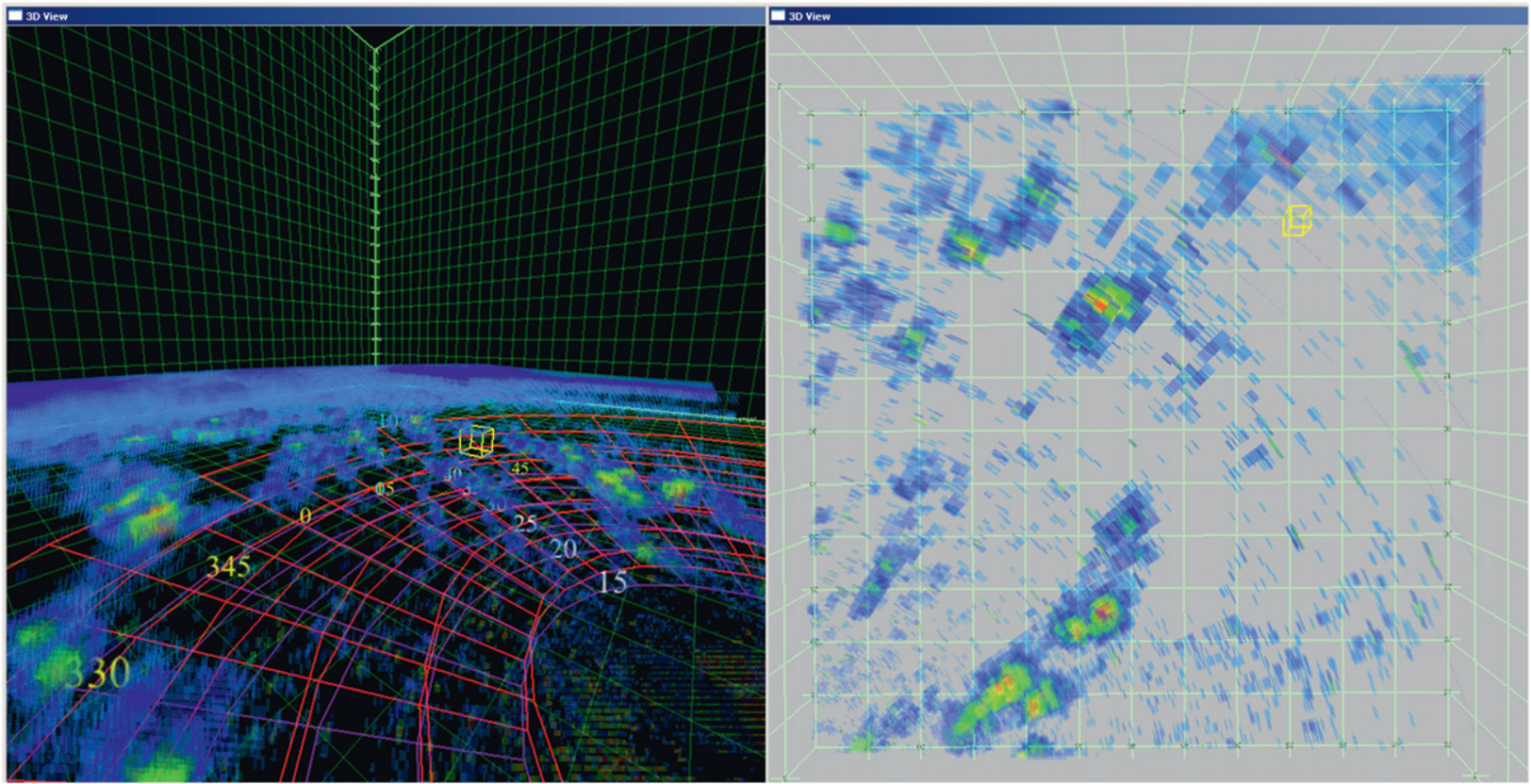

FIG. 2. Sample ARCA3D dual-3D view window depicting two perspectives of the same volume scan of $Z_{e}$. (left) Oblique view with polar grid showing range and azimuth scales. (right) View from above with Cartesian grid, zoomed in to a portion of the data space in the left view. In both views, the yellow-outlined cube is the 3D data probe. Cloud radar echoes are visible as the blue regions, and more dense/ more developed clouds are the brighter colors. A skin paint (e.g., Bringi et al. 1991) of the aircraft is visible just above the data probe in the right view.

geographic (latitude-longitude-altitude), relative Cartesian $(\mathrm{km}$, centered on the radar site), or polar (also centered on the radar site) coordinate extents. Userdefined Cartesian and polar grids can be overlaid onto the data in the 3D view. After setting transfer functions for at least one aircraft probe data variable, these data can be displayed in the system's 3D view along with the radar data (Fig. 3).

\section{b. Quantitative features}

Along with the visual presentation of collocated data for qualitative analysis, ARCA3D contains quantitative tools useful in determining correlations not easily made when using separate applications for each dataset. Because the view is a $2 \mathrm{D}$ representation of $3 \mathrm{D}$ space, an investigator cannot simply click on a point in a $3 \mathrm{D}$ view to learn the data values there (the location of a mouse cursor on the screen actually corresponds to a ray passing through the 3D space, so simple point-and-click interrogation will not work). A data probe (visible in Figs. 2 and 3 as a distinct $3 \mathrm{D}$ cube) that can be sized and moved throughout the $3 \mathrm{D}$ view space is necessary to select a portion of the data for quantitative analysis. The data probe displays values for all selected variables collocated at its center point, including the sample time, in its accompanying display window (Fig. 4). Desired variables from the data file(s) are listed in a user-configurable file. The data probe output also displays maxima, minima, and averages of each variable present from all points within the data probe's 3D space. Positioning and resizing the probe volume allow the investigator to surround any feature(s) of interest for which such information is desired. The data probe can also be locked onto the aircraft track contained in the displayed timeframe and scrolled along the track to display values of collocated radar and aircraft data points.

In addition to the information available from the data probe, 2D time series plots of two variables from either dataset can be generated along the aircraft flight track for a quick look (Fig. 5), for example, showing the change in some microphysical quantity as measured by the aircraft as it passes through the radar echo of multiple clouds. These 2D plots are generated through direct interaction of ARCA3D with MATLAB. Alternatively, variables from both datasets along the flight track can be exported to a text file that can be subjected to further analysis with other tools, such as spreadsheet or statistical software.

\section{c. Temporal collocation and cloud location adjustment}

Time is another important consideration in collocating radar and aircraft probe data. The aircraft is not always in the same place at the same time as the radar pulse, and if the two do exactly coincide temporally as 


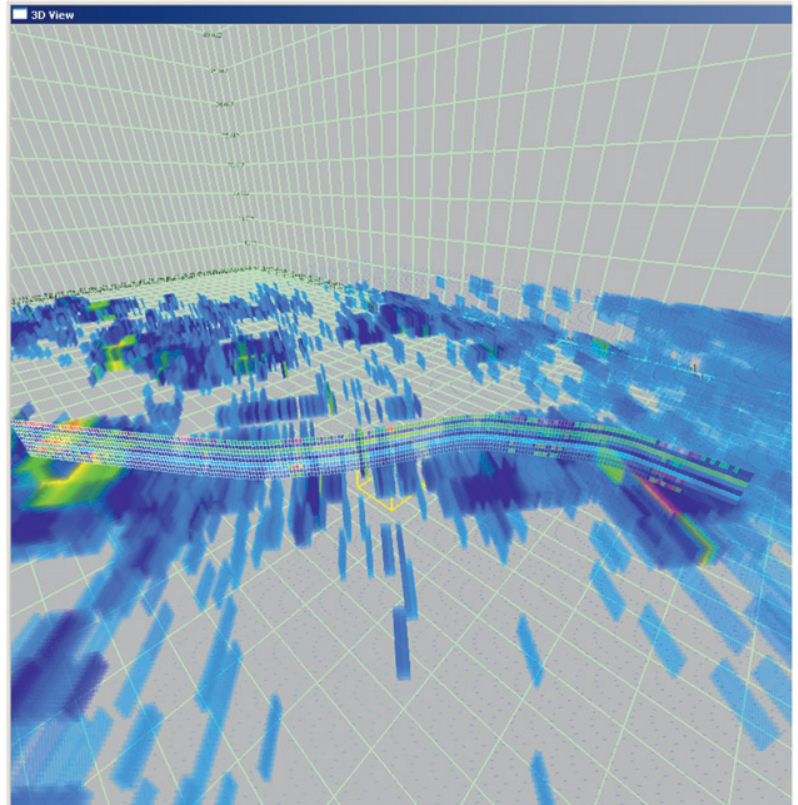

FIG. 3. Close-up of ARCA3D radar volume showing the aircraft track. Aircraft track corresponding to the time of the displayed radar volume scan is indicated by the multicolored ribbon near the center of the figure. Each color band on the ribbon represents a different user-defined aircraft data variable, with color changes indicating peaks based on that variable's transfer function. The bright-colored group of radar data points penetrated by the right end of the aircraft track is a skin paint. Aircraft travel was from right to left, indicated by increasing opacity values in that direction of the grid superimposed onto the aircraft track ribbon.

well as spatially, the radar values must be discarded as a skin paint [direct detection of the aircraft by the radar, e.g., Bringi et al. (1991), an example of which can be seen in the peak value of $Z_{e}$, shown in Fig. 5 and the bright colored radar cells near the right end of the aircraft track in Fig. 3] because it sees the aircraft rather than the cloud. To account for movement of radar echoes between the time the associated clouds were sampled by the aircraft and scanned by the radar, ARCA3D has a flexible mechanism to adjust for cloud movement. The user can input an adjustment time around which the radar data are moved, based on a user-input speed and direction as might be estimated from the prevailing environmental winds. Radar samples after this time are shifted along the direction of the wind, the distance depending on wind speed and the difference between the sample time and the adjustment time, and radar samples occurring before the adjustment time are shifted opposite the wind direction in a similar manner. This is admittedly a simplistic approach, and may introduce some location errors, but because the radar echo values evolve in time as well as in space, no data relocation scheme will achieve perfect results.

\section{d. Command query selection}

A command prompt is available for filtering the database in the structured query language (SQL). Filtering in this context is the method by which some data are excluded from view and calculations by selecting only those values that meet specific criteria. Filtering permits the user to select varying combinations and/or views of the data without actually affecting the data. Selection statements are entered in standard SQL syntax to filter the data within a defined set of spatial coordinates. Data located outside the chosen coordinates remain part of the working selection set, along with only those data inside the coordinates that were returned by the selection. Selection statements usually take the form of this example: "SELECT DBZ WHERE (DBZ>0.0) \& (ZDR >0.5) \& (ZDR<4.0)," which would select positive values of $Z_{e}$ at locations where $Z_{\mathrm{DR}}$ is between 0.5 and $4.0 \mathrm{~dB}$. Selections can be extended automatically, if desired, to other volumes upon loading so that repeating identical SQL statements is unnecessary. Such selections allow the user to omit undesired values or ranges of values from statistical calculations presented by the data probe, as well as from the 3D display if desired.

Once completed, query results can be viewed in $3 \mathrm{D}$ in context rendering mode. In this mode, the currently defined transfer function controls color and opacity of selected data, and nonselected data can still be viewed using the same colors of the transfer function but at a different opacity controlled by the user. Nonselected data are still visible after execution of a query (Fig. 6), which is often useful for orienting the user to where in the cloud or cloud field the query results are located. In this example, the opacity of nonselected data was set to $30 \%$, which is multiplied by the opacity of the current transfer function (90\%), resulting in an opacity of $27 \%$. The ability to view nonselected along with selected data can aid in keeping data in context, to see possible cloud edges, areas of fractocumulus, Bragg scattering, and other weak echoes.

\section{Application to RICO radar echo evolution}

Although conveyance of the utility of the new software is difficult in a $2 \mathrm{D}$ print medium, because its greatest advantages are in displaying the data in $3 \mathrm{D}$ and manipulating the view interactively, an initial application is presented here with a subset of the RICO data. The software allows shifting of one set of data relative to another to account for, say, the movement of the radar echoes downwind during the time an entire volume scan is collected. Wind data from the aircraft and dropsondes for each RICO day were used to center the $3 \mathrm{D}$ viewing of the radar data upon a central time for each radar volume, using skin paints of the aircraft by the radar as validation of these estimates. 


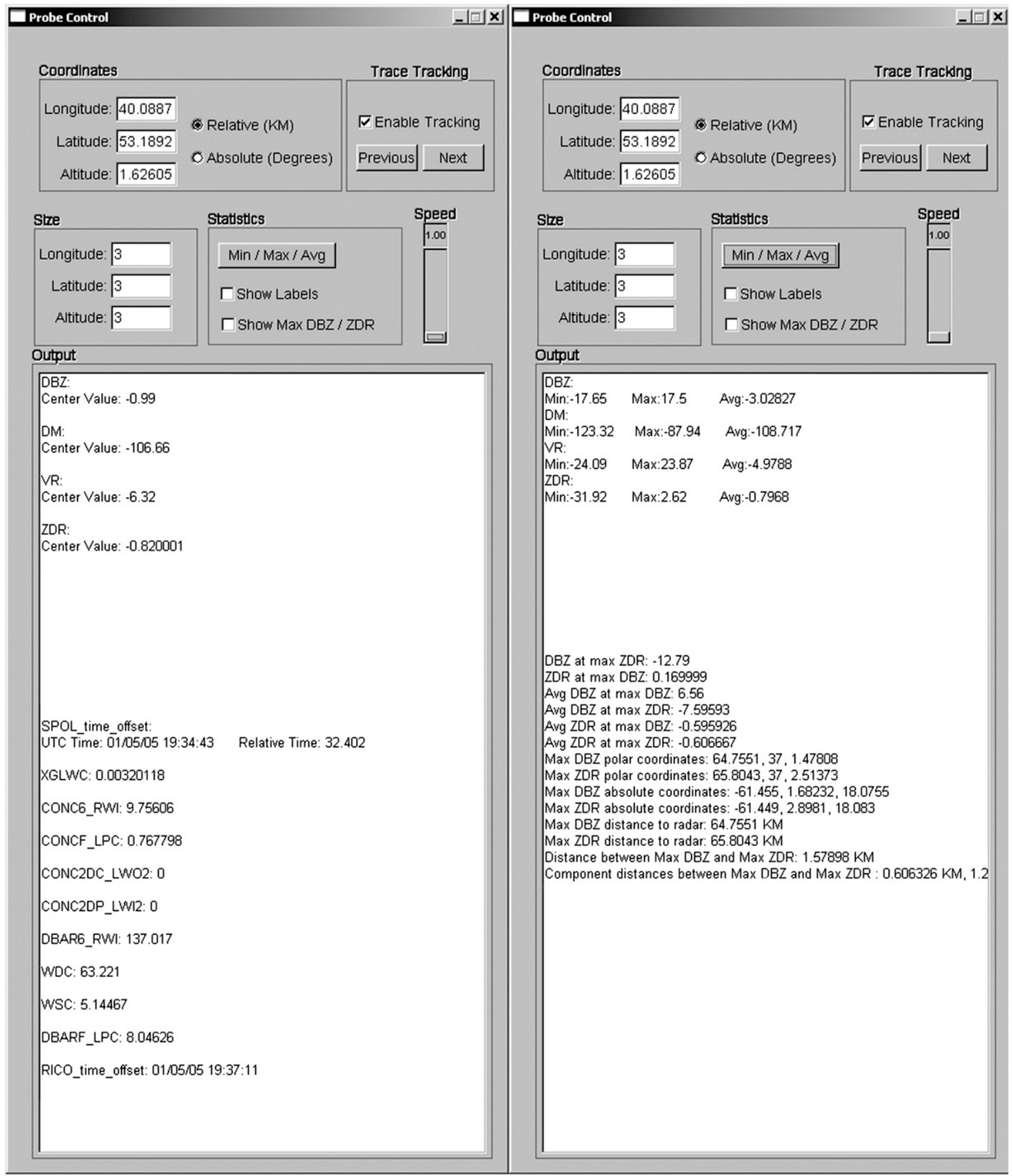

FIG. 4. Two views of ARCA3D data probe control window, displaying (left) values from both radar and aircraft datasets collocated at the data probe center and (right) minimum, maximum, and average values for radar data within the data probe, as well as values of $Z_{e}$ at the location of maximum $Z_{\mathrm{DR}}$ and vice versa, nearest-neighbor averages of each, and coordinates of the maxima. Control of data probe size, sampling resolution, and/or scroll speed is possible.

\section{a. Motivation}

Because the small trade wind cumulus clouds observed during RICO evolve and travel through the radar volume in time, the maximum $Z_{e}$ and $Z_{\mathrm{DR}}$ values (henceforth $Z_{e_{-} \max }$ and $Z_{\mathrm{DR} \_ \text {max }}$, respectively) detected in a particular cloud may not be collocated. Previous analyses of the RICO radar data by Knight et al. (2008) and Reiche and Lasher-Trapp (2010) noted that $Z_{e_{-} \max }$ was often not collocated with $Z_{\mathrm{DR} \_m a x}$ in the RICO data. In a study of Florida cumuli, Knight et al. (2002) speculated that separation of the greatest $Z_{e}$ and $Z_{\mathrm{DR}}$ signals in the cloud 


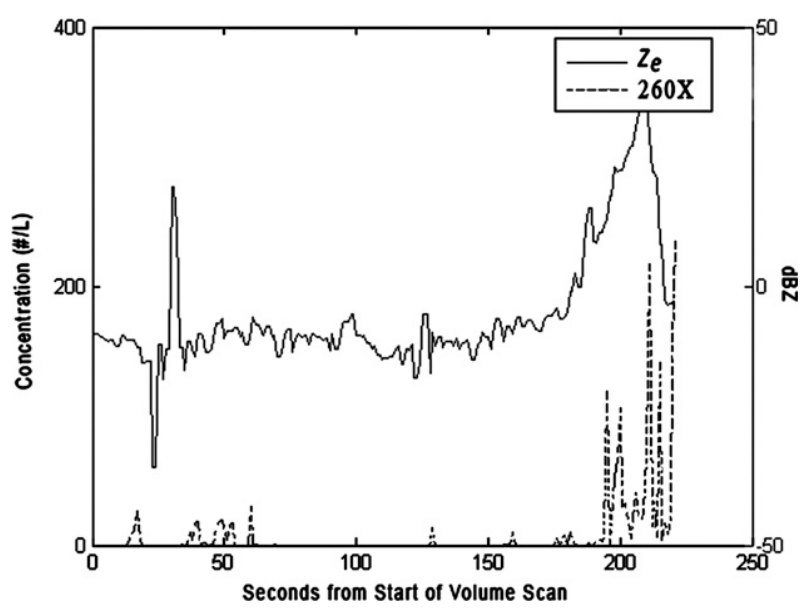

FIG. 5. Sample 2D plot of 1 aircraft probe variable (total droplet number concentration, 260X) and one radar variable $\left(Z_{e}\right)$ along the aircraft track for the duration of one radar volume scan. The peak toward the left on the solid curve plot is the same skin paint visible in Fig. 3. Peaks in the dashed curve correspond to 260X probe values also visible along the aircraft track ribbon in Fig. 3 (created via ARCA3D interface to MATLAB).

may result from larger drops appearing in weaker areas of clouds before coalescence begins in earnest, in regions often indistinguishable from Bragg scattering. Knight et al. (2008) referred to this phenomenon in the RICO data as size sorting, where the biggest drops fall out of the cloud faster because they have reached sizes too large to be carried higher in the cloud updrafts along with the smaller drops.

A possible source of these earliest, very large drops is giant aerosol particles (GA) such as sea salt (e.g., Woodcock 1953; de Leeuw 1986; Lewis and Schwartz 2004), which have diameters exceeding $2 \mu \mathrm{m}$ and thus enter the cloud as larger deliquesced drops than those having formed on smaller cloud condensation nuclei (CCN). Several modeling studies have suggested that GA can accelerate the formation of raindrops in cumuli because GA need little or no condensational growth prior to coalescence onset (e.g., Johnson 1982; Laird et al. 2000; Lasher-Trapp et al. 2001; Knight et al. 2002; Blyth et al. 2003). However, because smaller droplet number concentrations are present in maritime clouds, raindrop formation can occur quite quickly, even without the aid of GA (Feingold et al. 1999; Lasher-Trapp et al. 2008; Reiche and Lasher-Trapp 2010), leaving the importance of GA to precipitation formation in maritime clouds questionable. Observational studies are also in conflict regarding the importance of GA. Blyth et al. (2003) found GA were necessary to explain the early radar echo in small Florida cumuli over a range of $\mathrm{CCN}$ number concentrations, but other observational studies have shown that low CCN number concentrations may be the expla- nation for the quick development of precipitation in maritime clouds (Hudson and Yum 2001; Colón-Robles et al. 2006; Göke et al. 2007; Hudson et al. 2009).

The importance of GA to precipitation initiation in maritime clouds remains an open question, and the RICO dataset contains valuable information for studying this issue. However, compiling and synthesizing this information can be difficult with many current analysis tools. The remainder of this section will demonstrate ARCA3D's usefulness in studying the commonly separated $Z_{e_{-} \max }$ and $Z_{\mathrm{DR} \_ \text {max }}$ radar echoes in the RICO clouds, in assessing cloud-field characteristics, and in combining information regarding aerosol and droplet concentrations measured by the aircraft.

\section{b. RICO data analysis}

One of the greatest advantages of viewing radar data in $3 \mathrm{D}$ is the ease with which cases can be selected for study when a particular feature in the radar data is being explored, such as the separation of the $Z_{e}$ and $Z_{\mathrm{DR}}$ echo maxima. Ten days from the RICO campaign were chosen to study this radar echo evolution, because the clouds on these days were relatively isolated, shallow trade wind cumuli, well-sampled by the S-PolKa $10-\mathrm{cm}$ radar for their entire lifetime (staying within a range of $15-40 \mathrm{~km}$ ), and repeatedly penetrated by the C-130 aircraft. Using the new software, 15 clouds on each of the 10 days were used for quantitative analysis. The radar reflectivity was also filtered to minimize detection of nonmeteorological targets, such as frigate birds (e.g., Snodgrass 2006), and only values of $Z_{\mathrm{DR}}$ above $0.5 \mathrm{~dB}$ were considered above the level of noise [based on previous studies by Knight et al. (2008) and Reiche and Lasher-Trapp (2010)].

\section{1) CHARACTERISTICS OF THE CLOUD FIELD}

Cloud lifetime was evaluated for each of the 15 clouds over the 10 days studied, defined as the time from when the radar echoes first exhibited $Z_{e}>-8.0 \mathrm{~dB} Z$, to when $Z_{e}$ again dropped below that value. These values are within the reflectivity factor range where cloud drops may be indistinguishable from Bragg scattering, especially in small, trade wind cumuli (Knight and Miller 1993, 1998). Despite the likely presence of Bragg echoes, $-8.0 \mathrm{~dB} Z$ was used as the minimum $Z_{e}$ threshold only to define cloud lifetime and physical extent such as width and depth (no other data analysis was performed on values that may have been in the Bragg scattering regime).

Summaries of cloud width, depth, lifetime, and pulsing period were recorded using the resizing and positioning capabilities of the data probe and the Cartesian coordinate reference grid. Table 1 lists many of the statistics for each of the 10 days studied. Maximum cloud 

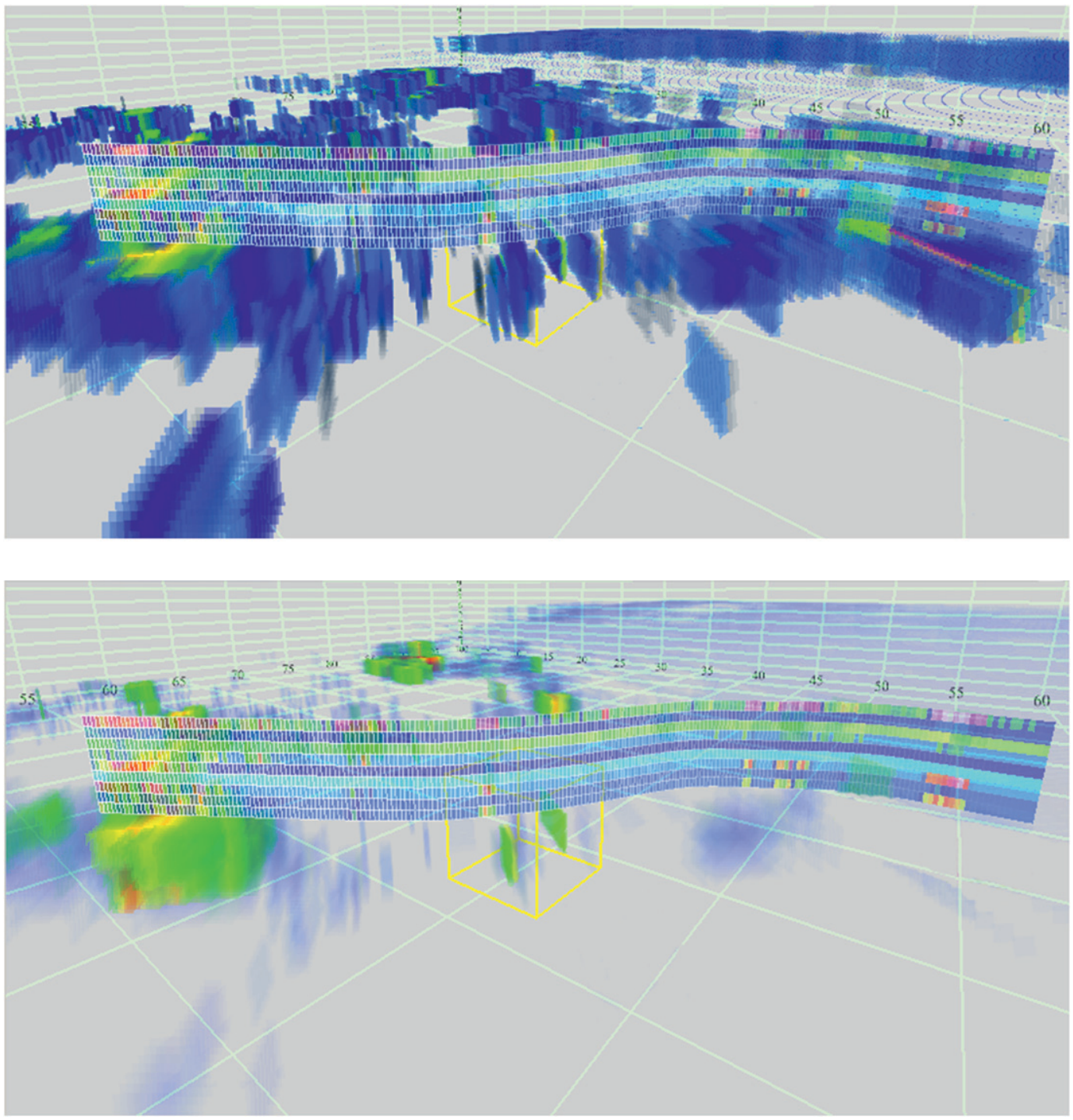

FIG. 6. A portion of an ARCA3D radar volume scan viewed (top) before and (bottom) after selection of data by a database query along with the C-130 aircraft flight path (ribbon) corresponding to the time of the volume scan. Transfer function values shown before execution of the query are $-8 \mathrm{dBZ}<Z_{e}<55 \mathrm{dBZ}$ ramped from $90 \%$ to $100 \%$ opacity. After selection of data by the query $0<Z_{e}<30 \mathrm{dBZ}$ and $Z_{\mathrm{DR}}>0.5 \mathrm{~dB}$, selected data remain at $90 \%-100 \%$ opacity, and unselected data are shown at $0 \%-5 \%$ opacity.

width ranged from 500 to $4000 \mathrm{~m}$, while maximum depth varied from 1000 to $2800 \mathrm{~m}$. Individual cloud lifetimes ranged from 20 min to well over $3 \mathrm{~h}$. Many of the RICO clouds exhibited a pulsing behavior similar to that seen in earlier studies of maritime and Florida cumuli (e.g., Saunders 1965; LeMone and Meitin 1984; French et al. 1999). However, mean pulse periods estimated from the radar data were often much longer than the 10-15 min observed in eastern Atlantic clouds by LeMone (1980). This discrepancy could be due to the pulse periods being observed through visual interpretation of radar echo evolution, the limited size of the dataset, or the presence of less vigorous updrafts and/or convergence in the specific clouds chosen for the current study.

\section{2) BEHAVIOR OF $Z_{E \_ \text {MAX }}$ AND $Z_{\text {DR_MAX }}$}

Over the cloud lifetime, characteristics of the $Z_{e \text { max }}$ and $Z_{\mathrm{DR} \_ \text {max }}$ of the cloud were noted by surrounding a chosen cloud echo region $\left(Z_{e}>-8.0 \mathrm{dBZ}\right)$ with the application's 3D data probe. The values and positions of $Z_{e \_ \text {max }}$ and $Z_{\mathrm{DR} \_ \text {max }}$ within the cloud and relative to each other were recorded as the cloud evolved through successive radar volumes. The ability to navigate both forward and backward in time through available volume 
TABLE 1. Characteristics of the 15 clouds over 10 RICO days used in this study.

\begin{tabular}{|c|c|c|c|c|c|c|c|c|c|}
\hline Flight & $\begin{array}{c}\text { Max } \\
\text { cloud } \\
\text { width }(\mathrm{m})\end{array}$ & $\begin{array}{c}\text { Max } \\
\text { cloud } \\
\text { depth }(m)\end{array}$ & $\begin{array}{l}\text { Mean echo } \\
\text { lifetime } \\
\text { (hh:mm) }\end{array}$ & $\begin{array}{c}\text { Mean echo } \\
\text { pulse period } \\
(\text { hh:mm })\end{array}$ & $\begin{array}{c}\text { Mean } \\
\text { lifetime } \\
Z_{e_{e} \max }(\mathrm{dB} Z)\end{array}$ & $\begin{array}{c}\text { Mean } \\
\text { lifetime } \\
Z_{\text {DR_max }}(\mathrm{dB})\end{array}$ & $\begin{array}{c}Z_{\mathrm{DR} \_m a x} \\
\text { start at } \\
\text { cloud } \\
\text { top }(\%)\end{array}$ & $\begin{array}{c}\text { GA } \\
\text { concentration } \\
\left(\mathrm{L}^{-1}\right)\end{array}$ & $\begin{array}{c}\text { Low-level } \\
\text { droplet } \\
\text { concentration } \\
\left(\mathrm{cm}^{-3}\right)\end{array}$ \\
\hline 10 Dec RF04 & 1300 & 1300 & 01:30 & $*$ & 38 & 1.7 & 73 & 75 & 46 \\
\hline 16 Dec RF06 & 3200 & 2500 & 01:20 & $00: 45$ & 52 & 0.9 & 47 & 165 & 84 \\
\hline 17 Dec RF07 & 2300 & 1100 & 02:00 & 01:00 & 28 & 1.2 & 80 & 64 & 44 \\
\hline 20 Dec RF09 & 500 & 1000 & $00: 45$ & 00:20 & 22 & 1.1 & 27 & 88 & 53 \\
\hline 5 Jan RF10 & 4000 & 2800 & $02: 45$ & $01: 25$ & 49 & 2.4 & 80 & 34 & 73 \\
\hline 11 Jan RF12 & 900 & 1150 & 00:50 & $00: 30$ & 33 & 1.0 & 33 & 122 & 83 \\
\hline 14 Jan RF14 & 1750 & 2000 & 02:00 & $*$ & 48 & 2.1 & 20 & 180 & 213 \\
\hline 19 Jan RF17 & 3550 & 2700 & $02: 20$ & 00:50 & 53 & 2.7 & 47 & 22 & 97 \\
\hline 23 Jan RF18 & 2400 & 1500 & 01:30 & 01:00 & 34 & 1.9 & 67 & 55 & 81 \\
\hline 24 Jan RF19 & 2800 & 2500 & 01:55 & $*$ & 38 & 2.1 & 80 & 60 & 86 \\
\hline Avg-all days & 2270 & 1855 & 01:41 & 00:50 & 40 & 1.7 & 55 & 87 & 86 \\
\hline
\end{tabular}

* No pulsing observed on these days.

scans with the new software was critical. Estimates of radar reflectivity factor and differential reflectivity calculated from microphysical probe data (Vivekanandan et al. 1991) collected by the C-130 aircraft were used when available to check radar values. The mean lifetime $Z_{e_{-} \max }$ for the 15 clouds varied by more than $30 \mathrm{~dB}$ across the 10 days studied, and the mean lifetime $Z_{\mathrm{DR} \_ \text {max }}$ varied by almost $2 \mathrm{~dB}$. Higher lifetime $Z_{e_{-} \text {max }}$ values tend to correspond to higher lifetime $Z_{\mathrm{DR} \_ \text {max }}$ values. Because $Z_{\mathrm{DR}}$ gives some indication of droplet size, this trend indicates that, in general, heavier precipitation consisted of larger drops.

Relative locations of $Z_{e_{-} \max }$ and $Z_{\mathrm{DR} \_ \text {max }}$ within a cloud through its lifetime were noted and tracked with the new visualization software and showed a tendency that may be unique to trade wind cumuli. The location of $Z_{e_{-} \max }$ would usually ascend and fall over the lifetime of the cloud in a predictable manner, rising with cloud growth and falling with cloud decay, corresponding to any observed pulsing in cloud development. In the clouds in this study, $Z_{e_{\text {max }}}$ was always at the cloud base by the end of the cloud lifetime, indicating descent of the most intense precipitation at that time. On the other hand, $Z_{\mathrm{DR} \_ \text {max }}$ initially was not collocated with $Z_{e \_ \text {max }}$, but the two maxima would become collocated near cloud base by the end of the cloud lifetime (e.g., Fig. 7). In some cases, $Z_{\mathrm{DR} \_ \text {max }}$ initially appeared much closer to cloud top than cloud base, whereas in other cases the $Z_{\mathrm{DR} \_ \text {max }}$ initially appeared much lower in the cloud. Figure 8 presents an example of the differences in locations of the two maxima during cloud lifetime for 5 January. Each line in the figure represents one cloud over its entire lifetime (normalized over chosen clouds for that day), and each data point a normalized relative distance from cloud base for the radar maximum, across each radar volume scan of the cloud. This analysis was completed for each of the 10
RICO days chosen for this study. The disparity in initial height of $Z_{e_{-} \text {max }}$ compared to $Z_{\mathrm{DR} \_ \text {max }}$ might be explained by the fact that initial values of $Z_{e_{-} \max }$ (using a minimum threshold of $-1 \mathrm{~dB} Z$, as in Knight et al. 2008) occurred 1-3 volumes earlier than the first appearance of initial values of $Z_{\mathrm{DR}_{\_} \max }$ above the noise threshold of $0.5 \mathrm{~dB}$. In all 15 of the clouds shown in Fig. 8, $Z_{\mathrm{DR} \_ \text {max }}$ occurs much lower in the cloud by the end of its lifetime, becoming collocated with $Z_{e_{-} \text {max }}$; this was characteristic of its behavior on other days as well. In Table 1 are the percentages of cases with $Z_{\mathrm{DR} \_ \text {max }}$ originating at cloud top on each day, and shows this to be the dominant tendency on some days but not others. Tracking this difference in the $Z_{D R \_m a x}$ echo evolution might reveal the importance of GA in raindrop formation because of its sensitivity to larger drops. The frequency with which $Z_{\mathrm{DR} \_ \text {max }}$ starts near cloud top is compared to other factors later in the study, including environmental GA number concentration, maximum cloud depth, and total cloud droplet number concentration to examine the progression of the warm-rain process in these clouds.

\section{3) DROP SIZE DISTRIBUTIONS WITHIN LOWER-LEVEL AND UPPER-LEVEL $Z_{\text {DR_MAX }}$}

In this section, two cases are presented in which the C-130 aircraft sampled the cloud within 1 min of seeing the $Z_{\mathrm{DR} \_ \text {max }}$ appear at that same location on radar or sampled an adjacent radar cell. These cases illustrate differences in the drops composing the $Z_{\mathrm{DR} \_ \text {max }}$ located at higher and lower regions of the cloud early in its lifetime.

Figures 9-11 depict a portion of the radar volume and C-130 aircraft track early in the cloud lifetime for a cloud on 5 January. In this cloud, $Z_{\mathrm{DR}_{\mathrm{m}} \max }$ began near cloud top (900 $\mathrm{m}$ at that time) and had descended to the C-130 flight level of $650 \mathrm{~m}$ during the volume shown. The drop 

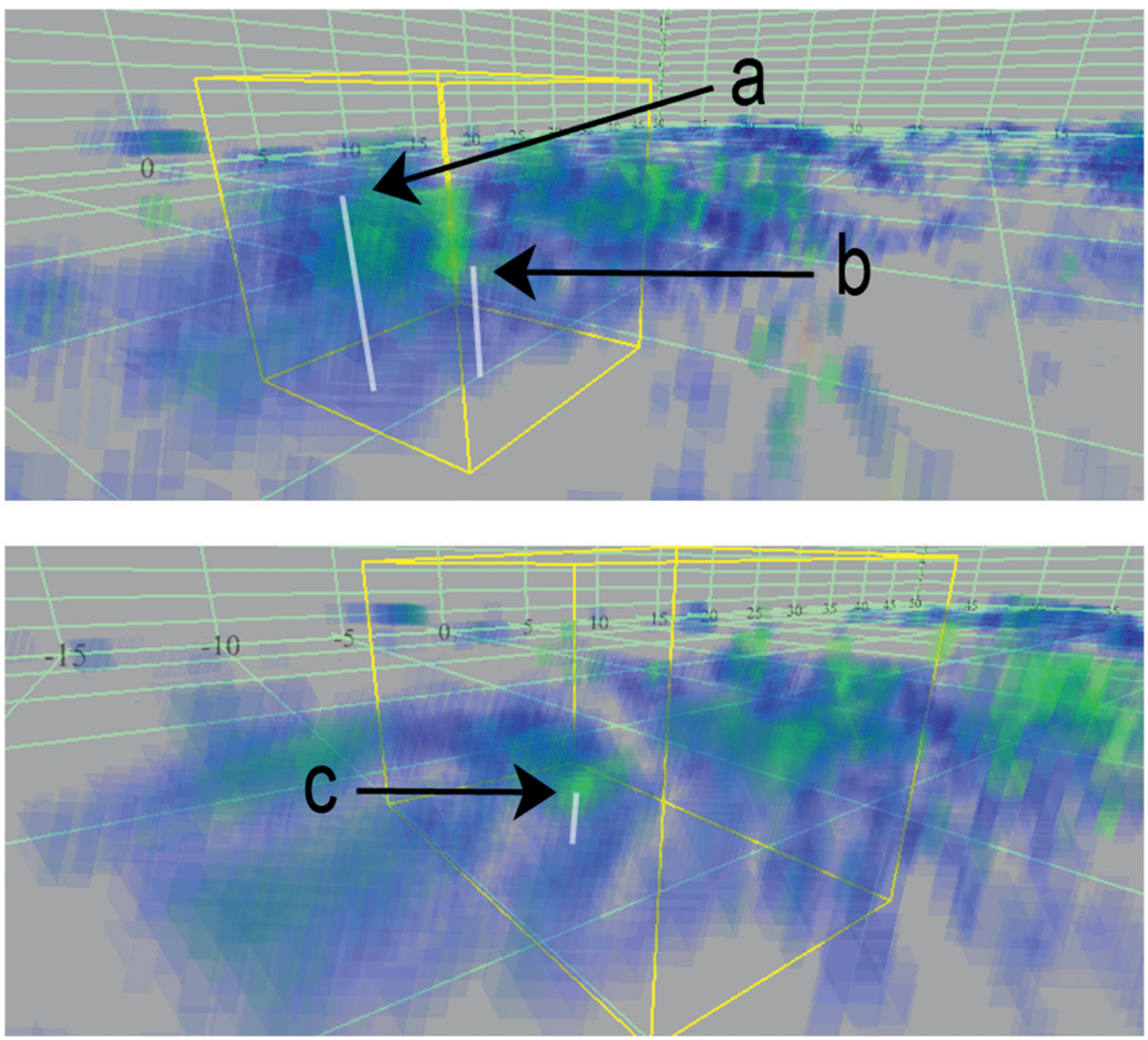

FIG. 7. ARCA3D volume scans (top) 2 and (bottom) 7 of 7 for a single cloud on $10 \mathrm{Dec}$ in the 3D visualization tool, showing locations of (b) $Z_{e_{-} \max }$ and (a) $Z_{\mathrm{DR} \_ \text {max }}$ at the top of thin white lines extending from the bottom of the probe space (highlighted by black arrows for clarity). Here, $Z_{\mathrm{DR} \_ \text {max }}$ begins near cloud top at $1200 \mathrm{~m}$. (c) The two maxima are collocated by end of cloud lifetime.

size distribution for drops greater than $100 \mu \mathrm{m}$ sampled by the C-130 drop probes is shown in Fig. 10, and two quick look $2 \mathrm{D}$ plots of $Z_{\mathrm{DR}}$ versus $2 \mathrm{DC}$ and versus $2 \mathrm{DP}^{3}$ probe total drop number concentration are shown in Fig. 11. These were used along with the data probe output and the 3D views in Fig. 9 to confirm radar and aircraft data collocation. Mean drop diameters and total drop number concentrations for drops $\geq 100 \mu \mathrm{m}$ and $\geq 1 \mathrm{~mm}$ diameter (Table 2) sampled by the aircraft indicate drops $\geq 1 \mathrm{~mm}$ for the 5 January case. In the 5 January cloud, the C-130 sampled the location of $Z_{\mathrm{DR} \_ \text {max }}(650 \mathrm{~m}$ above cloud base) $24 \mathrm{~s}$ after radar scanning. The theoretical value for $Z_{\mathrm{DR}_{-} \max }$ at this location is slightly lower than the value measured by the

\footnotetext{
${ }^{3}$ 2DC and 2DP Optical Array Probes manufactured by Particle Measurement Systems, Inc., Boulder, Colorado.
}

radar, and it appears to have a significant contribution from $1 \mathrm{~mm}$ or larger drops.

Figures 12-14 show similar views of data from a 10 December cloud. In this cloud, $Z_{\mathrm{DR} \_ \text {max }}$ first appeared near cloud base. It happened to be collocated with $Z_{e \text { max }}$ during this early volume, but the two maxima separated, becoming collocated again near the end of cloud lifetime. As shown in Table 2, the 10 December cloud did not have any drops $\geq 1 \mathrm{~mm}$ detected. Theoretical values for $Z_{\mathrm{DR}}$ from the drop size distributions for both clouds appear to be consistent with values measured by the radar. In the 10 December cloud, the actual $Z_{\mathrm{DR} \_ \text {max }}$ location was not penetrated by the C-130, but the adjacent radar sample at the same flight level $(50 \mathrm{~m}$ above cloud base) was, $57 \mathrm{~s}$ after radar scanning.

These two cases suggest an interesting relationship between the initial height of $Z_{\mathrm{DR} \_ \text {max }}$ and the drop size distribution. In the 5 January cloud, $Z_{\mathrm{DR} \_ \text {max }}$ first reached an 

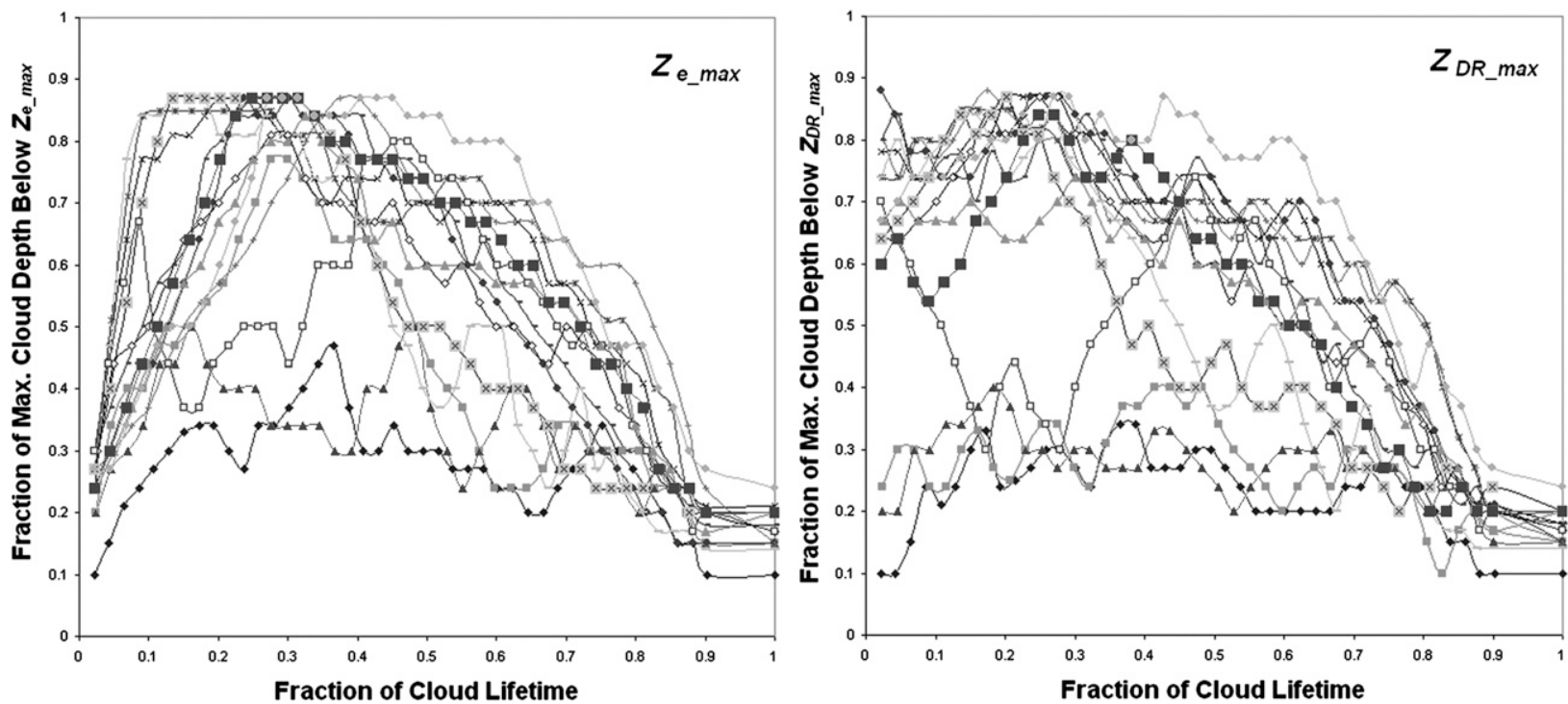

FIG. 8. Evolution of heights of observed (left) $Z_{e_{\_} \max }$ and (right) $Z_{\mathrm{DR} \_ \text {max }}$, normalized by cloud depth, over the normalized cloud lifetime for all 15 clouds studied for $5 \mathrm{Jan}$. This illustrates the differences in (right) initial $Z_{\mathrm{DR} \text { max }}$ locations relative to cloud base compared to (left) initial $Z_{e_{-} \max }$ locations. An obvious separation can be seen in initial relative $Z_{\mathrm{DR} \_ \text {max }}$ locations, which was apparent on the other chosen RICO days as well.

appreciable value closer to cloud top. While the C-130 also sampled this cloud early in its lifetime, it did so when the $Z_{\mathrm{DR} \text { max }}$ appeared to be descending, and the sample contained many more large drops than the 10 December cloud, including drops $\geq 1 \mathrm{~mm}$. Interestingly, the measured value of $Z_{\mathrm{DR} \text { max }}(1.9 \mathrm{~dB})$ was the lifetime maximum for this cloud-no later volume would have a $Z_{\mathrm{DR} \text { max }}$ greater than $1.7 \mathrm{~dB}$. This suggests that either these largest drops fell out of the cloud quickly and were no longer present in the next radar volume scan $(\sim 4 \mathrm{~min}$ later) or experienced drop breakup during descent. In the 10 December cloud, $Z_{\mathrm{DR} \_ \text {max }}$ began near cloud base. The aircraft sampled near $Z_{\mathrm{DR} \text { max }}$ early in the cloud lifetime and, although some large drops are present, none $\geq 1 \mathrm{~mm}$ were sampled. This is also reflected in the value of $Z_{\mathrm{DR} \text { max }}$ during this volume $(1.1 \mathrm{~dB})$, which was its largest so far in the cloud lifetime (it would reach a maximum lifetime value of $2.1 \mathrm{~dB}$ near end of life). In the next section, these tendencies will be examined with respect to ambient GA number concentrations and the number concentration of cloud droplets near the bases of the clouds.

\section{c. Importance of $G A$ versus $C C N$ to radar echo evolution}

Data from the forward scattering spectrometer probe ${ }^{4}$ (FSSP) on the C-130 were used to obtain GA number

\footnotetext{
${ }^{4}$ Manufactured by Droplet Measurement Technologies (DMT) Inc., Boulder, Colorado.
}

concentrations in the cloud environment (as in Reiche and Lasher-Trapp 2010) and cloud droplet number concentrations within the clouds for each of 10 RICO days. Observed GA number concentrations in clear air varied by an order of magnitude across the days examined, as did observed cloud droplet number concentrations (Table 1), providing an opportunity to find relationships among these variables and precipitation development as interpreted from observed radar echoes.

Comparing GA and drop number concentrations for the two days examined in the previous section, it can be seen that, of the days chosen for this study, 10 December had a fairly large GA number concentration and small drop number concentration, whereas 5 January had a small GA number concentration and greater drop number concentration. In the 10 December cloud, $Z_{\mathrm{DR} \text { max }}$ never ascended very far above cloud base; however, in the 5 January cloud, the low GA number concentration might have required drops to ascend closer to cloud top before producing appreciable $Z_{\mathrm{DR}}$ values.

Several different analyses of the RICO data (Hudson and Mishra 2007; Reiche and Lasher-Trapp 2010; Nuijens et al. 2009) have suggested that it is the cloud depth in these trade wind cumuli that tend to determine if precipitation will form in these clouds. Cloud depth may be related to precipitation formation through both the potentially longer time available for drops to grow and the greater overall amount of liquid water present in the cloud.

If a comparison of the height of the initial $Z_{\mathrm{DR}_{-} \max }$ versus GA number concentration is performed with 


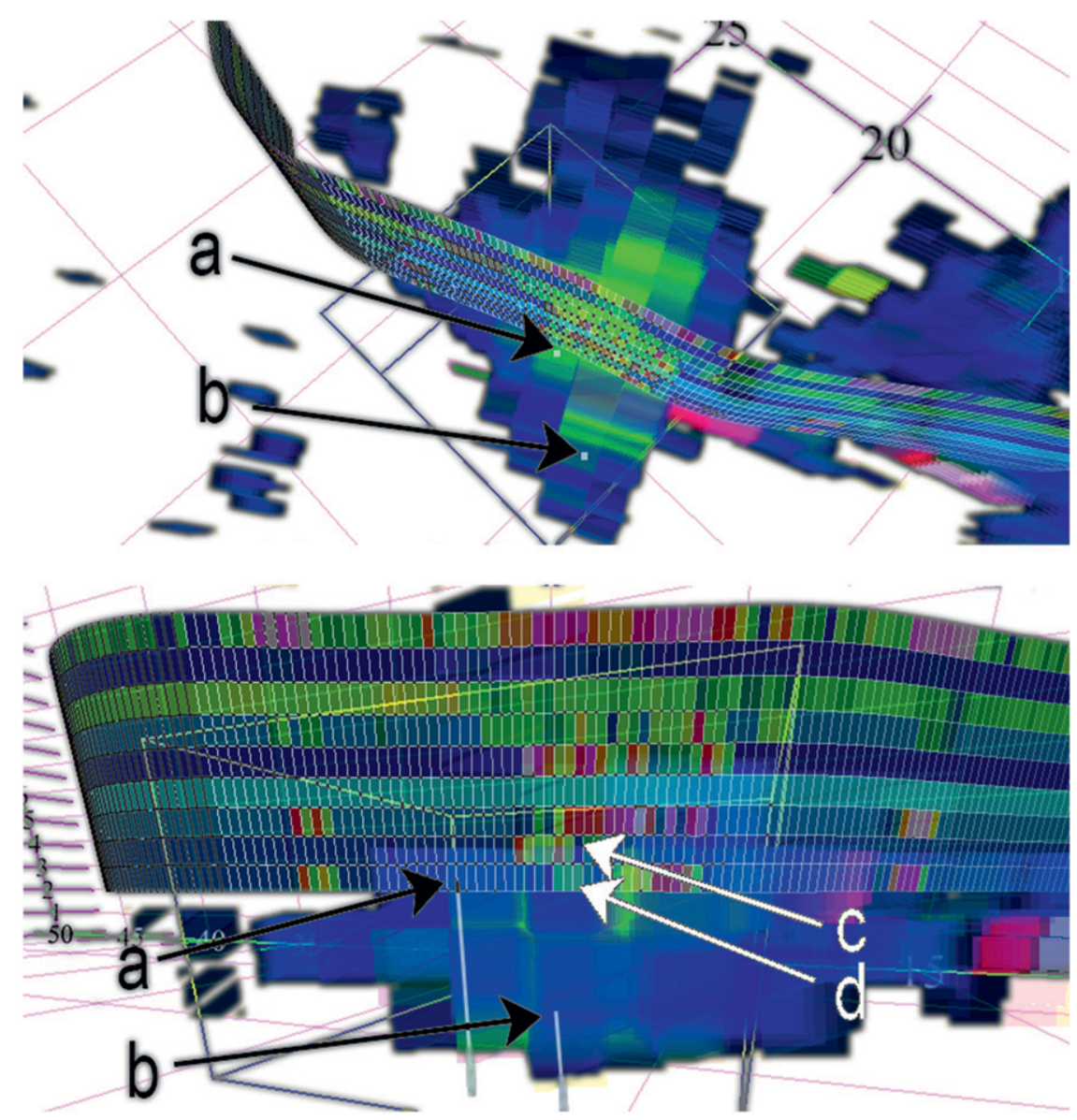

FIG. 9. ARCA3D view from (top) above and (bottom) side of a portion of the fifth volume of 23 in cloud lifetime for one cloud from $5 \mathrm{Jan}$ indicating locations of (a) $Z_{\mathrm{DR} \_ \text {max }}$ and (b) $Z_{e_{-} \max }$ (at tops of white poles extending from bottom of data probe space) and penetration by the C130 aircraft (ribbon) with peaks in (c) 2DP and (d) 2DC probe drop concentrations along the bottom two rows of ribbon (other aircraft data such as $260 \mathrm{X}$ concentration, liquid water content, and wind speed are also depicted on remaining rows). C-130 penetration was $650 \mathrm{~m}$ above cloud base, $24 \mathrm{~s}$ after radar scanning, collocated with $Z_{\mathrm{DR} \_ \text {max }}$, which first appeared closer to cloud top. Here, $Z_{\mathrm{DR} \_ \text {max }}=1.9 \mathrm{~dB}$, collocated with drop spectrum shown in Fig. $10 ; Z_{e_{-} \text {max }}=$ $32 \mathrm{~dB} Z$, lower in cloud; and $Z_{e}=9 \mathrm{~dB} Z$ at the location of $Z_{\mathrm{DR} \_ \text {max }}$. Theoretical values of $Z$ and $Z_{\mathrm{DR}}$ calculated from the drop spectrum in Fig. 10 at the location of $Z_{\mathrm{DR} \_ \text {max }}$ are $16 \mathrm{dBZ}$ and $1.7 \mathrm{~dB}$, respectively. Arrows and labels were not generated by the application and have been added for clarity.

a restriction of including only clouds from each day that grew to at least $75 \%$ of the maximum cloud depth for that day, a strong relationship between the percentage of $Z_{\mathrm{DR} \_ \text {max }}$ starting near cloud top and GA number concentration is evident $\left(R^{2} \approx 0.7\right)$. Although the choice of $75 \%$ threshold was somewhat arbitrary, it retained at least half of the clouds originally analyzed on each day. Applying a threshold that is a percentage of maximum cloud depth may be more representative of environmental conditions for each day than using an absolute depth threshold.

Using the same cloud-depth threshold (at least $75 \%$ of the maximum for the day) for initial $Z_{\mathrm{DR} \_ \text {max }}$ percentage near cloud top versus cloud droplet number concentration also leads to a strong correlation (Fig. 15). The 3 days with the highest observed GA number concentrations (>100 L ${ }^{-1}: 16$ December, 11 January, and 14 January) can be seen along the bottom of the figure. The remaining days have a very high correlation between droplet number concentration and $Z_{\mathrm{DR} \_ \text {max }}$ occurring at cloud top $\left(R^{2} \approx 0.8\right)$. This plot suggests that GA may be a controlling factor in the initial location of $Z_{\mathrm{DR} \text { max }}$ when there are more than $100 \mathrm{~L}^{-1}$ present because the cloud droplet number concentrations for those days were also relatively high, which tends to slow coalescence onset. On the remaining days, GA may be responsible for the early $Z_{\text {DR_max }}$ echoes appearing lower in these deeper clouds, 


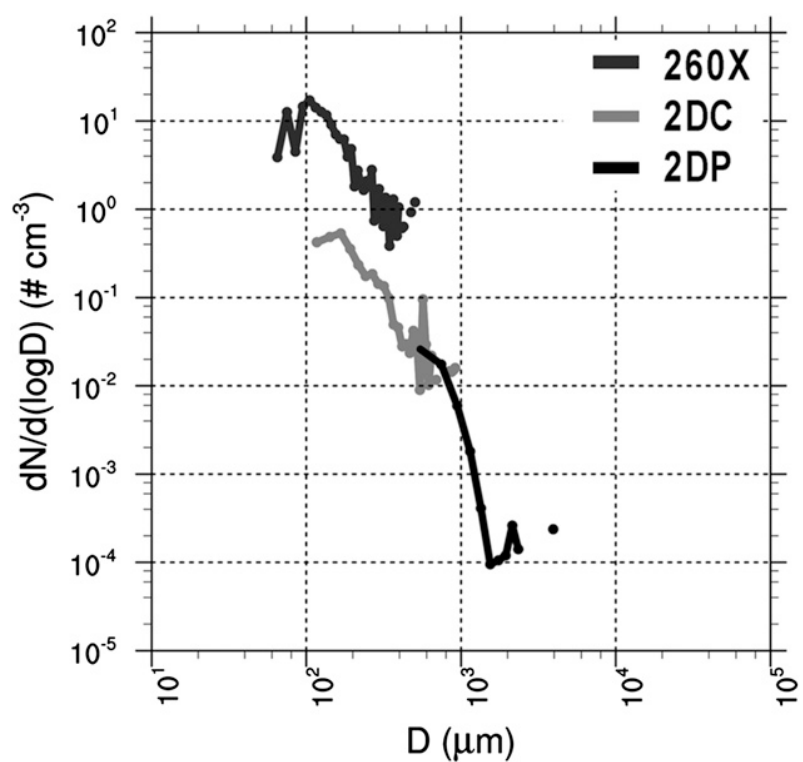

FIG. 10. Drop spectrum from 260X, 2DC, and 2DP probes on C-130 for 5 Jan cloud penetration collocated with radar data depicted in Fig. 9 (1859:54-1900:00 UTC, width of radar cell equivalent to $7 \mathrm{~s}$ of the $\mathrm{C}-130$ flight).

but as shown in Fig. 15 the GA were anticorrelated with cloud droplet number concentrations. Either fewer GA or greater cloud droplet number concentrations slow the warm-rain process_-additional time/liquid water content is required for coalescence to proceed in earnest and produce precipitation sized drops. In these cases it appears reasonable that appreciable values for $Z_{\mathrm{DR} \text { max }}$ do not appear until closer to cloud top. From this dataset and analysis it is difficult to discern, however, if GA or cloud droplet number concentrations dominate the early $Z_{\mathrm{DR} \text { max }}$ echo when GA number concentrations are less than $100 \mathrm{~L}^{-1}$.

\section{Summary and discussion}

Using ARCA3D with radar and aircraft data from the RICO field campaign, the evolution of the warm-rain process within trade wind cumulus clouds over their lifetimes has been investigated and the possible role of GA in the formation of warm rain has been explored. A novel approach has been used for examination of polarimetric radar data, involving investigation of the volume maxima in radar reflectivity $\left(Z_{e_{-} \max }\right)$ and differential reflectivity $\left(Z_{\mathrm{DR} \_ \text {max }}\right)$, their positions within cloud and relative to each other, and how those factors evolved over cloud lifetimes. This approach has been avoided in past studies (e.g., Knight et al. 2008; Reiche and Lasher-Trapp 2010) because both maxima have been difficult for investigators to track and quantify with
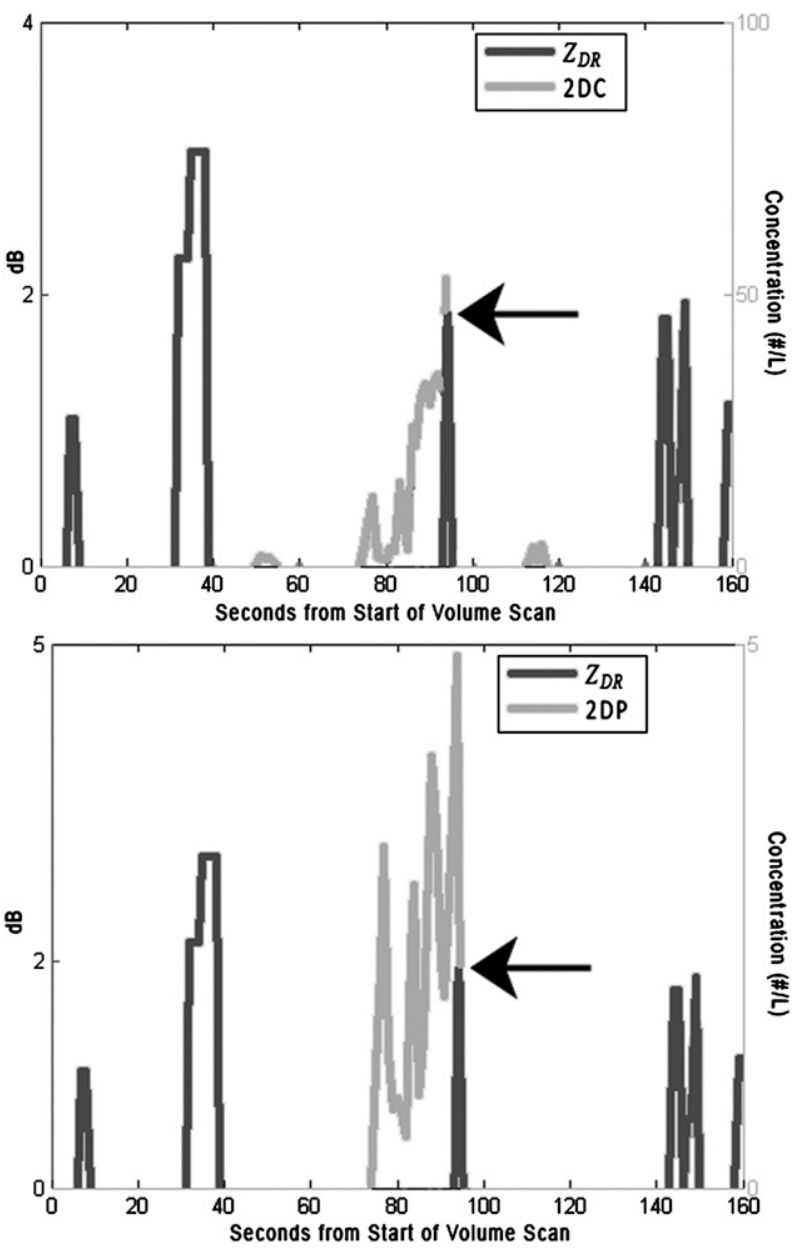

FIG. 11. 2D plots along the C-130 flight path from 5 Jan volume scan in Figs. 9 and 10. Arrows (not generated by the application) indicate $Z_{\mathrm{DR} \text { max }}$ in chosen cloud (values $<0.5 \mathrm{~dB}$ filtered). (top) $2 \mathrm{DC}$ number concentration and $Z_{\mathrm{DR}}$. (bottom) 2DP number concentration and $Z_{\mathrm{DR}}$ (created via ARCA3D interface to MATLAB)

currently available software. The main findings of this study, using ARCA3D, were the following:

- Cloud depth appears to modulate the formation of precipitation in the trade wind cumuli, consistent with other studies of the RICO data.

- In considering only the deepest clouds observed on each day, a strong positive correlation $\left(R^{2} \approx 0.7\right)$ exists between the initial $Z_{\mathrm{DR} \_ \text {max }}$ occurring low in the cloud and GA number concentration. The larger sizes of GA enable the formation of large drops quickly, and therefore low in the cloud and appear to dominate the initial $Z_{\mathrm{DR} \text { max }}$, if present in sufficient number concentrations.

- Also in the deepest clouds, an initial $Z_{\mathrm{DR} \_ \text {max }}$ appearing higher in the cloud was positively correlated $\left(R^{2} \approx 0.8\right)$ with cloud droplet number concentration when cases with $\mathrm{GA}>100 \mathrm{~L}^{-1}$ were excluded. This is 
TABLE 2. Comparison of $Z_{\mathrm{DR}}$ values from radar and C-130 probe spectra.

\begin{tabular}{|c|c|c|c|c|c|c|c|}
\hline & & \multicolumn{4}{|c|}{ C-130 drop probe $(260 \mathrm{X}, 2 \mathrm{DC})$ data } & & \\
\hline & & \multicolumn{2}{|c|}{$\geq 100 \mu \mathrm{m}$} & \multicolumn{2}{|c|}{$\geq 1 \mathrm{~mm}$} & \multicolumn{2}{|c|}{$Z_{\mathrm{DR}}(\mathrm{dB})$} \\
\hline \multicolumn{2}{|c|}{ Flight } & $N\left(\mathrm{~L}^{-1}\right)$ & $\bar{D}(\mu \mathrm{m})$ & $N\left(\mathrm{~L}^{-1}\right)$ & $\bar{D}(\mu \mathrm{m})$ & $\begin{array}{c}\text { Measured } \\
\text { from S-PolKa }\end{array}$ & $\begin{array}{l}\text { Calculated from } \\
\text { C-130 probe DSD }\end{array}$ \\
\hline $10 \mathrm{Dec}$ & RF 04 & 332.0 & 343.2 & 0.0 & - & 0.80 & 1.10 \\
\hline 5 Jan & RF 10 & 3522.0 & 202.7 & 2.0 & 1375.9 & 1.90 & 1.70 \\
\hline
\end{tabular}

consistent with longer growth times being necessary to initiate rain by the warm-rain process when greater cloud droplet number concentrations are present. This effect is only seen, however, when too few GA were present to dominate the initial $Z_{\mathrm{DR}}$ echo.
These results may at first appear contradictory to those of Reiche and Lasher-Trapp (2010), who concluded that GA only have a minor importance in rain formation in trade wind cumuli observed during RICO. The emphasis in those studies, however, was the evolution of the
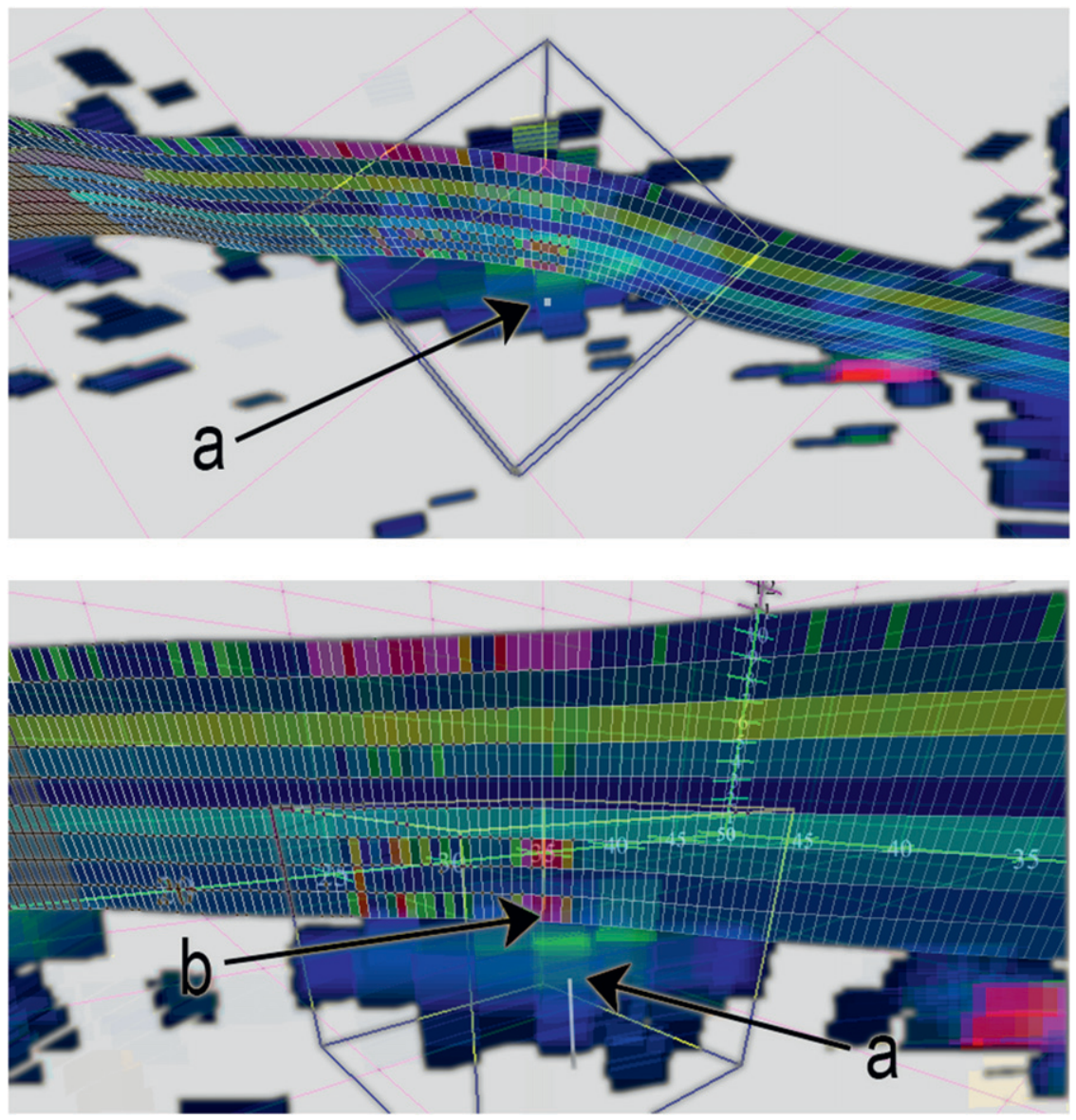

FIG. 12. As in Fig. 9, but for a portion of the third volume of 13 in cloud lifetime for one cloud from 10 Dec. Here, (top) $Z_{e_{-} \max }$ and $Z_{\mathrm{DR} \_ \text {max }}$ are collocated. C-130 penetration was $50 \mathrm{~m}$ above cloud base, $57 \mathrm{~s}$ after radar scanning, with (bottom) 2DC probe peak drop number concentration of $4.91 \mathrm{~L}^{-1}$ adjacent to radar cell with $Z_{e_{-} \max }$ and $Z_{\mathrm{DR} \_ \text {max }}$, both of which first appeared closer to cloud base (the two maxima separated in later volumes during ascent and became collocated again near cloud base by end of cloud lifetime). Here, $Z_{\mathrm{DR}_{-} \max }=1.1 \mathrm{~dB}$ and $Z_{e_{-} \max }=13.92 \mathrm{~dB} Z$, and $Z_{\mathrm{DR}}=0.8 \mathrm{~dB}$ and $Z_{e}=11.6 \mathrm{~dB} Z$ in the adjacent radar cell collocated with the $2 \mathrm{DC}$ probe peak. Theoretical values of $Z$ and $Z_{\mathrm{DR}}$ calculated from the drop spectrum in Fig. 13 at the location of the $260 \mathrm{X}$ probe peak are $14.3 \mathrm{dBZ}$ and $1.1 \mathrm{~dB}$, respectively. 


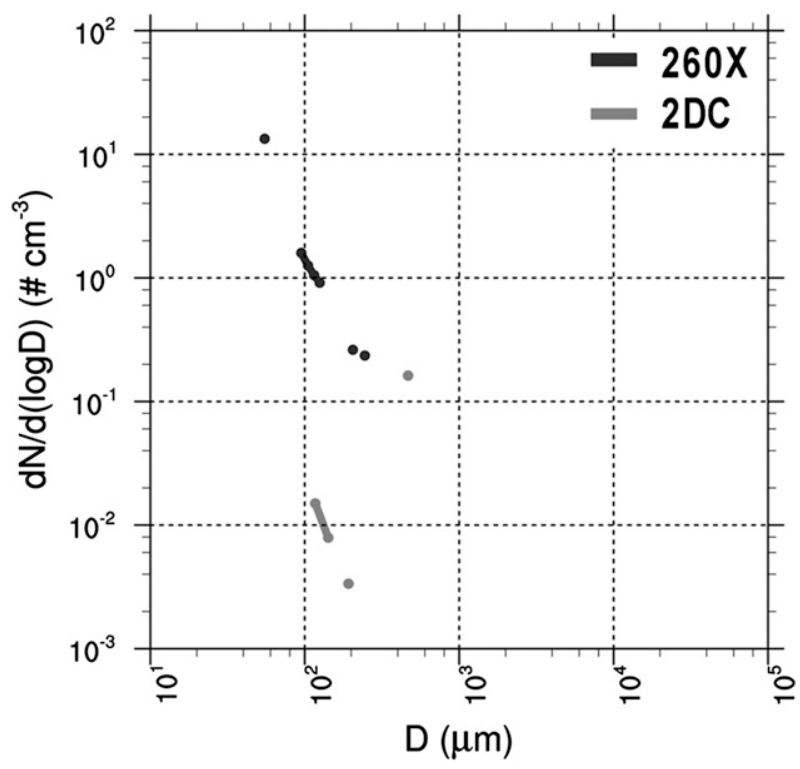

FIG. 13. Drop spectrum from $260 \mathrm{X}$ and 2DC probes (no drops registered on 2DP for this cloud) on the C-130 for $10 \mathrm{Dec}$ cloud penetration depicted in Fig. 12 (1814:51-1814:57 UTC, width of radar cell equivalent to $7 \mathrm{~s}$ of the $\mathrm{C}-130$ flight).

maximum reflectivity echo in the clouds, following the evolution of substantial precipitation formation. In the present study, the evolution of the earliest maximum differential reflectivity echoes was emphasized to focus on formation of the first raindrops in the cloud, which may or may not contribute to any significant precipitation produced later that would dominate the maximum reflectivity echo evolution. Here, when GA are present in number concentrations exceeding $100 \mathrm{~L}^{-1}$, they do appear to be important for the formation of the earliest raindrops, but cannot be assumed to be important for the bulk of the precipitation produced in the cloud. The numerical modeling of Reiche and Lasher-Trapp (2010) suggests that they would not be.

The tracking of the evolution of the spatial locations of the maximum reflectivity and differential reflectivity echoes performed in this study do support the observation of such behavior noted by Knight et al. (2008) and provide evidence that this behavior may be related to the microphysical evolution of the cloud. Knight et al. (2008) suggested that the earliest differential reflectivity echoes result from size sorting of the biggest droplets in the cloud, apart from the region of the cloud within which significant precipitation may develop later, and the analysis here also supports that speculation.

Use of ARCA3D was invaluable in obtaining these results. The ability to view a large amount of both radar and aircraft data simultaneously and interactively in 3D, seeing entire volume scans of clouds rather than consecutive azimuthal sweeps, volume rendering of values

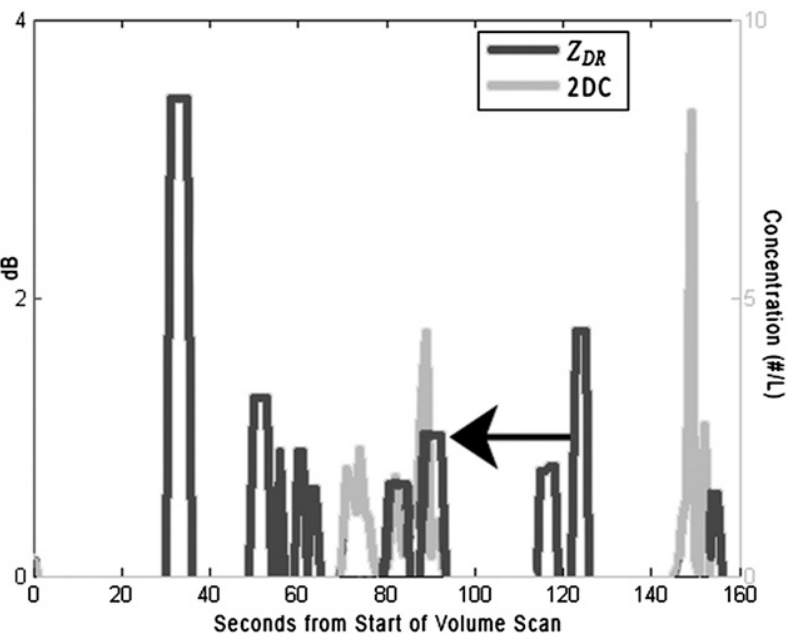

FIG. 14. 2D plot along the C-130 flight path from 10 Dec volume scan in Figs. 12 and 13. Arrow (not generated by the application) indicates $Z_{\mathrm{DR}}$ adjacent to $Z_{\mathrm{DR} \text { max }}$ in chosen cloud (created via ARCA3D interface to MATLAB)

rather than isosurfaces, flexible filtering and querying, and statistical output of the configurable 3D data probe all allowed reasonably efficient exploration of the 150 clouds examined for the current study. Still images presented herein cannot adequately represent the advantages of the software because a user can interact directly with three-dimensional views of the data, adjusting perspective (viewing angle, zoom, pan, etc.) and altering color and opacity values in real time to get the best view.

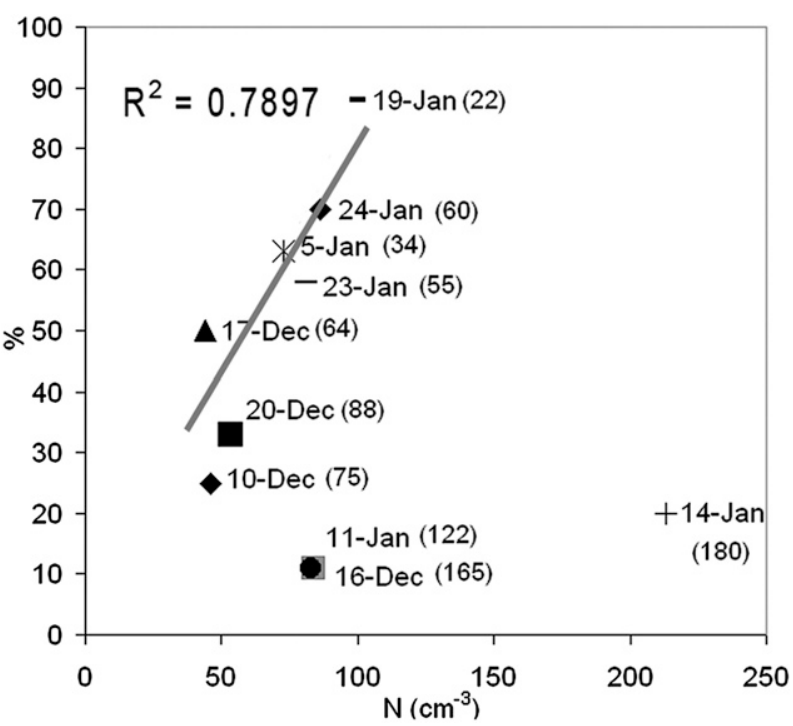

FIG. 15. Percentage of $Z_{\mathrm{DR} \text { max }}$ starting near cloud top vs cloud droplet number concentration for clouds each day that reached at least $75 \%$ maximum cloud depth for that day. Correlation shown calculated without 16 Dec, 11 Jan, or 14 Jan. Numbers in parentheses by each day indicate GA number concentrations $\left(\mathrm{L}^{-1}\right)$. 
Such interactive visual tools hold great promise for many applications in atmospheric science, and further development of ARCA3D is planned, with eventual dissemination to the atmospheric science community.

Acknowledgments. The authors wish to acknowledge NCAR for the use of the Solo II software package, and Robert Rilling and Scott Ellis of the NCAR/Earth Observing Laboratory for assistance with the NCAR $\mathrm{S}$-PolKa radar data. We would also like to thank the participants of RICO for their data collection efforts, Amanda Sheffield for statistical analysis of C-130 data from each RICO flight, and other members of the Purdue Cloud Microphysics Group and the Purdue Rendering and Perceptualization Laboratory (PURPL) for numerous helpful discussions and suggestions. Support for this project was provided by National Science Foundation Grant IIS-0513464. NCAR is also funded by the National Science Foundation.

\section{REFERENCES}

Arthur, D. K., 2009: Radar and aircraft observations of precipitation evolution in trade wind cumuli. M.S. thesis, Dept. of Earth and Atmospheric Sciences, Purdue University, 125 pp.

Beard, K. V., and H. T. Ochs III, 1993: Warm-rain initiation: An overview of microphysical mechanisms. J. Appl. Meteor., 32, 608-625.

Blyth, A. M., S. G. Lasher-Trapp, W. A. Cooper, C. A. Knight, and J. Latham, 2003: The role of giant and ultragiant nuclei in the formation of early radar echoes in warm cumulus clouds. J. Atmos. Sci., 60, 2557-2572.

Bringi, V. N., D. A. Burrows, and S. M. Menon, 1991: Multiparameter radar and aircraft study of raindrop spectral evolution in warm-based clouds. J. Appl. Meteor., 30, 853-880.

Carneiro, B., C. Silva, and A. Kaufman, 1996: Tetra-Cubes: An algorithm to generate 3D isosurfaces based upon tetrahedra. Proc. Ninth Brazilian Symp. on Computer Graphics and Image Processing, Rio de Janeiro, Brazil, Brazilian Computer Society, 205-210.

Clyne, J., P. Mininni, A. Norton, and M. Rast, 2007: Interactive desktop analysis of high-resolution simulations: Application to turbulent plume dynamics and current sheet formation. New J. Phys., 9, 301.

Colón-Robles, M., R. M. Rauber, and J. B. Jensen, 2006: Influence of low-level wind speed on droplet spectra near cloud base in trade wind cumulus. Geophys. Res. Lett., 33, L20814, doi:10.1029/ 2006GL027487.

de Leeuw, G., 1986: Vertical profiles of giant particles close above the sea surface. Tellus, 38B, 51-61.

Ellis, S. M., J. Vivekanandan, S. Goeke, E. A. Brandes, J. Stith, and R. J. Keeler, 2001: In-situ verification of remote aircraft icing detection using S-band polarization radar measurements. Preprints, 30th Int. Conf. On Radar Meteorology, Munich, Germany, Amer. Meteor. Soc., 2B.6. [Available online at http:// ams.confex.com/ams/30radar/techprogram/paper_21989.htm.]

Feingold, G., W. R. Cotton, S. M. Kreidenweis, and J. T. Davis, 1999: The impact of giant cloud condensation nuclei on drizzle formation in stratocumulus: Implications for cloud radiative properties. J. Atmos. Sci., 56, 4100-4117.
French, J. R., G. Vali, and R. D. Kelly, 1999: Evolution of small cumulus clouds in Florida: Observations of pulsating growth. Atmos. Res., 52, 143-165.

Göke, S., H. T. Ochs III, and R. M. Rauber, 2007: Radar analysis of precipitation initiation in maritime versus continental clouds near the Florida coast: Inferences concerning the role of CCN and giant nuclei. J. Atmos. Sci., 64, 3695-3707.

Hibbard, B., J. Kellum, B. Paul, D. Santek, and A. Battaiola, cited 2009: Vis5D documentation. [Available online at http://vis5d. sourceforge.net/doc/.]

Hudson, J. G., and S. S. Yum, 2001: Maritime-continental drizzle contrasts in small cumuli. J. Atmos. Sci., 58, 915-926.

, and S. Mishra, 2007: Relationships between CCN and cloud microphysics variations in clean maritime air. Geophys. Res. Lett., 34, L16804, doi:10.1029/2007GL030044.

— S. Soble, V. Jha, and S. Mishra, 2009: Correlations of small cumuli droplet and drizzle drop concentrations with cloud condensation nuclei concentrations. J. Geophys. Res., 114, D05201, doi:10.1029/2008JD010581.

Johnson, D. B., 1982: The role of giant and ultragiant aerosol particles in warm-rain initiation. J. Atmos. Sci., 39, 448-460.

Keeler, R. J., J. Lutz, and J. Vivekanandan, 2000: S-Pol: NCAR's polarimetric Doppler research radar. Proc. IGARSS-2000, Honolulu, HI, Institute of Electrical and Electronics Engineers, $1570-1573$.

Knight, C. A., and L. J. Miller, 1993: First radar echoes from cumulus clouds. Bull. Amer. Meteor. Soc., 74, 179-188. , and - 1998: Early radar echoes from small, warm cumulus: Bragg and hydrometeor scattering. J. Atmos. Sci., 55, 2974 2992.

, J. Vivekanandan, and S. G. Lasher-Trapp, 2002: First radar echoes and the early $Z_{D R}$ history of Florida cumulus. J. Atmos. Sci., 59, 1454-1472.

_ L. J. Miller, and R. A. Rilling, 2008: Aspects of precipitation development in trade wind cumulus revealed by differential reflectivity at S band. J. Atmos. Sci., 65, 2563-2580.

Laird, N. F., H. T. Ochs, R. M. Rauber, and L. J. Miller, 2000: Initial precipitation formation in warm Florida cumulus. J. Atmos. Sci., 57, 3740-3751.

Lasher-Trapp, S. G., C. A. Knight, and J. M. Straka, 2001: Early radar echoes from ultragiant aerosol in a cumulus congestus: Modeling and observations. J. Atmos. Sci., 58, 35453561.

— S. Anderson-Bereznicki, A. Shackelford, C. H. Twohy, and J. G. Hudson, 2008: An investigation of the influence of droplet number concentration and giant aerosol particles upon supercooled large drop formation in wintertime stratiform clouds. J. Appl. Meteor. Climatol., 47, 2659-2678.

Lawrence Livermore National Laboratory, cited 2009: CF metadata: NetCDF Climate and Forecast (CF) metadata convention. [Available online at http://cf-pcmdi.llnl.gov/.]

LeMone, M. A., 1980: The marine boundary layer. Proc. Workshop on the Planetary Boundary Layer, Boulder, CO, Amer. Meteor. Soc., 182-231.

, and R. J. Meitin, 1984: Three examples of fair-weather mesoscale boundary layer convection in the tropics. Mon. Wea. Rev., 112, 1985-1997.

Lewis, E. R., and S. E. Schwartz, 2004: Sea Salt Aerosol Production. Amer. Geophys. Union, 413 pp.

McCaslin, P., P. McDonald, and E. Szoke, cited 2009: Developing and testing a $3 \mathrm{D}$ visualization workstation application at FSL. Earth System Research Laboratory. [Available online at http:// laps.noaa.gov/d3d/ams99/paper/Developing_D3D.html.] 
Nuijens, L., B. Stevens, and A. Pier Siebesma, 2009: The environment of precipitating shallow cumulus convection. J. Atmos. Sci., 66, 1962-1979.

Oye, R., C. Mueller, and S. Smith, 1995: Software for radar translation, visualization, editing, and interpolation. Preprints, 27th Conf. on Radar Meteorology, Vail, CO, Amer. Meteor. Soc., 359-361.

Paluch, I. R., and C. A. Knight, 1986: Does mixing promote cloud droplet growth? J. Atmos. Sci., 43, 1994-1998.

Rauber, R. M., and Coauthors, 2007: Rain in shallow cumulus over the ocean-The RICO campaign. Bull. Amer. Meteor. Soc., 88, 1912-1928.

Reiche, C., and S. Lasher-Trapp, 2010: The minor importance of giant aerosol to precipitation development within small tradewind cumuli observed during RICO. Atmos. Res., 95, 386-399.

Rinehart, R. E., 2004: Radar for Meteorologists. Rinehart Publishing, $482 \mathrm{pp}$.

Saunders, P. M., 1965: Some characteristics of tropical marine showers. J. Atmos. Sci., 22, 167-175.

Snodgrass, E. R., 2006: Precipitation characteristics of trade wind clouds during RICO derived from radar, satellite, and aircraft measurements. M.S. thesis, Dept. of Atmospheric Sciences, University of Illinois at Urbana-Champaign, $101 \mathrm{pp}$.

Space Science and Engineering Center, cited 2009: McIDAS: Man computer interactive data access system. University of Wisconsin-Madison. [Available online at http://www.ssec.wisc. edu/mcidas/.]

Unidata Program Group, cited 2009a: Unidata's integrated data viewer. University Corporation for Atmospheric Research. [Available online at http://www.unidata.ucar.edu/software/ idv/docs/userguide/.]

, cited 2009b: NetCDF. University Corporation for Atmospheric Research. [Available online at http://www.unidata. ucar.edu/software/netcdf/.]

Vincenty, T., 1975: Direct and inverse solutions of geodesics on the ellipsoid with application of nested equations. Survey Rev. $X X I I$, 176, 88-93.

Vivekanandan, J., W. M. Adams, and V. N. Bringi, 1991: Rigorous approach to polarimetric radar modeling of hydrometeor orientation distributions. J. Appl. Meteor., 30, 1053-1063.

Woodcock, A. H., 1953: Salt nuclei in marine air as a function of altitude and wind force. J. Meteor., 10, 362-371. 